\title{
LA PINTURA DE LO COTIDIANO EN LA FRANCIA DEL SIGLO XVIII. CREACIÓN Y TRANSFORMACIÓN DEL TÉRMINO «PINTURA DE GÉNERO» EN LA HISTORIOGRAFÍA ANGLOAMERICANA: DEL REALISMO A LO COTIDIANO
}

\author{
The Painting of Everyday Life in Eighteenth-Century \\ France. Creation and Transformation of the Term \\ "Genre Painting" in Anglo-American Historiography: \\ from Realism to Everyday Life
}

Jaime BLANCO APARICIO

Universidad Complutense de Madrid

jaimeblapa@gmail.com

Fecha de recepción: 29/09/2017

Fecha de aceptación definitiva: 10/09/2018

RESUMEN: Al estudiar la pintura francesa del siglo XVIII a través de la historiografía angloamericana de los años 90, nos topamos a menudo con diversos términos, tales como pintura de género, de lo cotidiano o realista, que parecen ser utilizados de forma indistinta. Sin embargo, tras estos usos subyacía el deseo de construir un nuevo modelo interpretativo sobre esta pintura a partir de la noción de cotidianidad, que simplemente reactualizaba los imaginarios y discusiones -políticas y estéticasde los siglos XIX y XX, recogidas, a su vez, en los Cultural Studies y en la New Art History. Animaban, así, a mirar aquella pintura en función de problemáticas ajenas a las representaciones propias del siglo XVIII, impidiendo, finalmente, su correcta comprensión. Proponemos, por tanto, una aproximación historiográfica que busca comprender la transformación de la noción de pintura de género en pintura de lo cotidiano, poniendo de manifiesto la ideología subyacente a esta. 
Palabras clave: pintura francesa; siglo XVIII; pintura de género; pintura de lo cotidiano; realismo; New Art History.

ABSTRACT: When studying French painting of the eighteenth-century through Anglo-American historiography of the 90s, we often encounter various terms, such as genre painting, everyday life or realistic, which seem to be used indistinctly. However, behind these uses underlay the desire to build a new interpretative model of the French painting of the eighteenth century from the notion of everyday life, which simply updated the imaginary and discussions -politics and aestheticsof the nineteenth and twentieth century, collected, also, in the Cultural Studies and the New Art History. They encouraged us, therefore, to look at that painting in terms of problems unrelated to the typical representations of the eighteenth century, preventing, finally, their correct understanding. We propose, therefore, a historiographical approach that will seek to understand the transformation of the notion of genre painting into everyday life painting, highlighting the underlying ideology.

Key words: French Painting; Eighteenth-Century; Genre Painting; Everyday Life Painting; Realism; New Art History.

Al estudiar la pintura francesa del siglo XVIII a través de la historiografía angloamericana de los años 90, nos topamos a menudo con diversos términos, tales como pintura de género, pintura de lo cotidiano o pintura realista. Empleados a menudo de forma indistinta, mediante su uso no se buscaría tanto describir un tipo de pintura opuesta a la pintura de historia, tal y como se entendía en el siglo XVIII (adscrita al problema de la supuesta jerarquía de los géneros establecida por André Félibien) ${ }^{1}$, sino, más bien, describir un fenómeno artístico novedoso, fundamental para comprender las transformaciones culturales que estaban teniendo lugar en la sociedad dieciochesca, que permitiría iluminar el devenir histórico posterior. El presente artículo no afrontará, por tanto, ni la cuestión de la jerarquía de los géneros ${ }^{2}$, ni tampoco se detendrá en si la pintura de género realmente

1. FÉlibien, André. Conférences de l'Académie Royale de Peinture et de Sculpture pendant l'année 1667. Ámsterdam: Estienne Roger, 1706, p. 16.

2. Wrigley, Richard. The French Art Criticism, from the Ancien Régime to the Restoration. Oxford: Clarendon Press, 1993, pp. 285 y s. Kirchner, Thomas. "La nécessité d'une hiérarchie des genres". Revue d'Esthétique. La naissance de la théorie de l'art en France, 1640-1720, 1997, 31/32, pp. 187-196. DÉmoris, René. "La hiérarchie des genres en peinture de Félibien aux Lumières". En RoQUE, Georges (dir.). Majeur ou mineur? Les hiérarchies en art. Nimes: Jacqueline Chambon, 2000, pp. 53-66. LEDBURY, Mark. "The Hierarchy of Genre in the Theory and Practice of Painting in Eighteenth-Century France». En DÉcultot, Élisabeth y LEDBury, Mark (eds.). Théories et débats esthétiques au dix-Huitième siècle. Éléments d'une enquête. París: Honoré Champion, 2001, pp. 233-252. ANDERMAN, Barbara. "La notion de peinture de genre à l'époque de Watteau». En RAMADE, Patrick y EIDELBERG, Martin (coms.). Watteau et la fête galante. París: RMN, 2004, pp. 29-43. ANDERMAN, Barbara. "Félibien and the Circle 
supuso un fenómeno novedoso en la Francia del dieciocho. Su objetivo principal será analizar desde un punto de vista historiográfico las transformaciones que se producen en el significado del término pintura de género desde finales del siglo XVIII hasta finales del siglo XX, a medida que los nuevos debates artísticos se suceden -como el realismo- y a medida que los acontecimientos históricos y políticos -como el auge del socialismo- condicionan aquellos. Dentro de este proceso, la pintura de género se irá adscribiendo a ciertos debates, tales como el realismo o lo cotidiano, que transformarán su sentido original, incorporando nuevos significados ajenos al siglo XVIII. A través de este breve recorrido genealógico se buscará, asimismo, poner de manifiesto la ideología subyacente a las definiciones empleadas por la historiografía angloamericana para describir la pintura de género francesa del siglo XVIII como pintura de lo cotidiano, revelándose, tras ello, sus profundas deudas respecto a las corrientes de izquierda del siglo XIX y XX, las cuales han condicionado, a su vez, las nuevas aproximaciones a la Historia del Arte.

Si bien algunos historiadores anglosajones tienden a prefigurar en la pintura de género francesa del siglo XVIII los debates sobre el realismo y lo cotidiano elaborados en el siglo XIX y XX, apoyándose para ello en los propios textos del siglo XVIII, donde supuestamente se afirmaría la irrupción de este nuevo fenómeno artístico de lo cotidiano; sin embargo, un análisis detallado de las fuentes dieciochescas revela, si no una lectura errónea, al menos interesada por parte de aquellos. Un fenómeno que es reconocido abiertamente en las dos principales exposiciones que fundamentan este nuevo sentido dado a la pintura de género en el mundo angloamericano: la exposición de 1997 titulada Intimates Encounters ${ }^{3}$ y la exposición de 2003 The Age of Watteau, Chardin, and Fragonard 4 . El propio Richard Rand expresaba en su prefacio su deseo de alejarse del significado que pudiera tener el término en el siglo XVIII, para emplear la noción de pintura de género en un sentido que define como sentido moderno, esto es, adscrito a las escenas de la vida cotidiana: "Genre painting (in the modern sense of the termthat is, pictures of everyday life) $)^{5}$. De una forma similar se expresará el programa de mano de la exposición de Washington de 2003:

In eighteenth-century France, the term peinture de genre (genre painting) suggested any type of painting that was not history painting. Depicting serious themes drawn from history, literature, or the Bible, history painting had been considered the

of Colbert: A Reevaluation of the Hierarchy of Genres». En Donald, Diana y O'Gorman, Frank (eds.). Ordering the World in the Eighteenth Century. Londres: Palgrave-Macmillan, 2005, pp. 143-162.

3. RAND, Richard (com.). Intimates Encounters. Love and Domesticity in Eighteenth-Century France. Hanover: Hood Museum of Arte-Darmouth College, 1997.

4. Bailey, Colin B.; Conisbee, Philip y Gaehtgens, Thomas W. (coms.). The Age of Watteau, Chardin, and Fragonard. Masterpieces of French Genre Painting. Washington, Ottawa, Berlin, New Haven: National Gallery of Art, National Gallery of Canada, Staaliche Museen zu Berlín, Yale University Press, 2003.

5. RAND, Richard. "Preface and Acknowledgments». En RAND. Intimates Encounters..., p. X. 
highest aspiration of the artist since this theory was developed during the Renaissance some three hundred years earliers... Thus the official hierarchy of subject matter categorized as "genre» other types of painting -such as landscapes, still lifes, hunting scenes, portraits, or even scenes from daily life. However, in modern times (and thus in our exhibition) the definition of genre painting has narrowed and now designates scenes of daily life, be they real or imaginary ${ }^{6}$.

Ambas exposiciones señalaban claramente su intención de adscribir el término pintura de género a las escenas de la vida cotidiana, dando con ello una respuesta a la moda que sobre lo cotidiano estaba teniendo lugar en el mundo académico norteamericano. El catálogo de Washington admitía, asimismo, que, a pesar del poco reconocimiento concedido por la Academia dieciochesca a esta pintura de las escenas de género (en el sentido de escenas de la vida cotidiana), sin embargo, se trataba de uno de los fenómenos artísticos más originales del periodo: "Despite the lowly position adscribed to genre painting by the Academy during the eighteenth century, subjects from everyday life engaged some of the most original and gifted artists of the Ancien Régime» ${ }^{7}$. Bailey consideraba, igualmente, que a pesar de la gran variedad de términos existentes en el siglo XVIII para referirse a ella ${ }^{8}$, sin embargo, su artículo -y el conjunto de la exposición- tenía como pretensión justificar la existencia de una pintura de género entendida como escenas de la vida cotidiana. No obstante, y ante la ausencia de un reconocimiento terminológico en el siglo XVIII, Bailey incidía en que eran las propias formas artísticas las que afirmarían y justificarían este uso. Ambas exposiciones proponían, así, una aproximación formalista ${ }^{9}$ sustentada sobre un relato formal que buscaba ofrecer una imagen unitaria del arte del siglo XVIII, que se iniciaba con las fêtes galants de Watteau y que culminaba con Boilly, poniendo en relación artistas muy diversos entre sí gracias a un denominador común: lo cotidiano.

Lo cotidiano permitía descubrir en la pintura dieciochesca una serie de principios ocultos que estaban determinando subterráneamente las transformaciones y dinámicas tanto artisticas como sociales del siglo. Por un lado, lo popular y, por otro lado, y derivado de lo anterior, la dialéctica y las tensiones sociales, que tanto lo cotidiano como lo popular permitían comprender. En este sentido, la emergencia de la burguesía y las tensiones sociales que ello generó, manifestado a través del conflicto entre representaciones y gustos artísticos, se convertían para esta historiografía angloamericana en el quid de la cuestión para comprender las

6. Citamos el programa de mano de la exposición de Washington de 2003 recogido en la review que de la exposición realizó SCHroder, Anne L. "Review: The Age of Watteau, Chardin, and Fragonard». Eighteenth-Century Studies, 2004, 37, 3, pp. 455-463.

7. Bailey, Colin B.; Conisbee, Philip y Gaehtgens, Thomas W. "Curator's Preface and Acknowledgements». En Bailey, ConisBee y Gaehtgens. The Age of Watteau..., p. VIII.

8. BaIley, Colin B. "Surveying Genre in Eighteenth-Century French Painting». En BAILEY, ConISBeE y Gaehtgens. The Age of Watteau..., pp. 2-4.

9. Ledbury, Mark. "Review: Age of Watteau, Chardin, and Fragonard». The Art Book, 2004, 11, 3. 
transformaciones culturales del siglo XVIII, siendo las escenas de género o de lo cotidiano lo que permitía tomar conciencia histórica sobre tal cambio. La emergencia de la clase burguesa como consecuencia de las transformaciones económicas; la conciencia popular y la lucha de clases; y la emergencia de un gusto por la realidad; características del materialismo dialéctico, se veían reformuladas a través del término cotidiano, que ponía el acento sobre las representaciones culturales, esto es, sobre las formas de vida. Una reinterpretación del realismo social a través de lo cotidiano que se vio favorecida por los Cultural Studies, los cuales recuperaban, a su vez, los debates hegeliano-marxistas de los años 30 que inspirados por Lukács se desarrollaron principalmente en Francia.

Para Richard Rand el género era algo más que una simple temática entre otras posibles. Era una especie de fuerza energizante que dinamizaba el siglo, transformando las formas artísticas en su conjunto, revelando los sentidos ocultos y las dialécticas que dinamizan la sociedad del dieciocho: "Genre painting, however, was no longer the energizing force ${ }^{10}$. De forma similar, se expresaba el ensayo de Martin Schieder en el catálogo de Washington, quien interpretaba el género como una fuerza que penetraba en las otras temáticas pictóricas, convirtiéndolas en manifestaciones de género: "To what extent did the representational modes and content of genre painting affect other painting genre -mythology, religious history, portaiture? Why were more and more painters in all genres accentuating the everyday, the human element, and true-to-life depictions? ${ }^{11}$. Para Schieder esa fuerza invisible del género era, a su vez, la manifestación de una dinámica más profunda que hundía sus raíces en lo social, que todo lo transformaba. Igualmente, esta energía fue subrayada por Timothy Rub, director del Hood Museum of Art, vinculando, además, la pintura de género con las transformaciones sociales y políticas: "Development of genre painting during a period of remarkable social and political change, and within this increasingly important artistic category, the growing prominence of themes related to matters of the heart and the home» ${ }^{12}$. Para Barbara Gaehtgens esta fuerza dinamizadora provenía claramente de lo burgués: «No one could have described more clearly the current paradigm shift -away from admiring the sublimity of a dramatic historical event, to being touched by the genuine and naive emotions expressed in a depiction of bourgeois society ${ }^{13}$. En su artículo, lo cotidiano era un claro reflejo de las profundas transformaciones culturales que estaban sucediendo en la familia, en el rol de lo femenino, en la infancia, en el comercio, etc.; tal y como también subrayaba Thomas W. Gaehtgens

10. RAND, Richard. "Love, Domesticity, and the Evolution of Genre Painting in EighteenthCentury France». En RAND. Intimates Encounters..., p. 16.

11. SCHIEDER, Martin. "Sorti de son genre": Genre Painting and Boundary Crossing at the End of the Ancien Régime». En Bailey, Conisbee y Gaehtgens. The Age of Watteau..., p. 63.

12. RuB, Timothy. "Foreword». En Intimates Encounters..., p. VII.

13. Gaentgens, Barbara. "The Theory of French Genre Painting and its European Context». En Bailey, Conisbee y Gaehtgens. The Age of Watteau..., p. 53. 
con el epígrafe "Genre Painting and Social Reality» con el que da comienzo su artículo ${ }^{14}$. En este se planteaban cómo a través de las escenas de género se podría penetrar en la realidad cultural de la época: "Recent studies illuminate the cultural and social background of the times, providing a historical fabric for the content found in these painting [...] Doubtless, the painting present a certain social reality, or an artistic version of it, but they were bought by people who themselves lived a very different reality» ${ }^{15}$. Si bien fueron las transformaciones sociales las que favorecieron el ascenso de la burguesía, motor a su vez del cambio económico, social, político y estético, de igual modo, para esta historiografía, la burguesía tenía una inclinación hacia aquella realidad donde ellos se sentían reconocidos -como había señalado Hadjinicolaou- ${ }^{16}$, favoreciendo, finalmente, una pintura de lo cotidiano. En este sentido y siguiendo la obra de Crow, Thomas Gaehtgens planteaba que fue gracias al Salón -un espacio de intercambio cultural- que la burguesia adquiere conciencia de la necesidad de unas formas que la representen en sus valores, tal y como se reflejaría en la pintura de género: «In this sense, genre painting answered the bourgeoisie's need for qualities that were lacking in the noble aspirations and elevated emotions of classical history painting", pues "genre's manifold themes and styles satisfied widely divergent interests» $y$, por tanto, "genre painting contributed to the social metamorphosis that ocurried during the Age of Enlightenment; in some ways, it was also a product of this proces, which has such far-reaching historical and intellectual consequences» ${ }^{17}$.

En ambas exposiciones esta interpretación de lo cotidiano se justificaba a través de la supuesta influencia de la pintura holandesa del siglo XVII en el arte francés del siglo XVIII, lo que constituirá otro de los tópicos de la historiografía dieciochesca $^{18}$. Convertida la pintura holandesa, a lo largo del siglo XIX y XX, en

14. Gaehtgens, Thomas W. "Genre Painting in Eighteenth-Century Collections». En Bailey, CONisbee y Gaehtgens. The Age of Watteau..., p. 78.

15. Ibid., p. 78 .

16. «L'effet esthétique n'est pas autre chose que le plaisir qu'éprouve le spectateur qui se reconnaît dans l'idéologie imagée de l'œuvre. Pour l'historien, les tâches qui résultent de l'existence de cette reconnaissance sont de taille: l'analyse de l'idéologie imagée d'une œuvre particulière se fondera sur l'histoire de son appréciation". Hadjinicolaou, Nicos. Histoire de l'art et lutte de classes. París: Maspero, 1973 , p. 195.

17. Gaehtgens, Thomas W. "Genre Painting in Eighteenth-Century Collections». En Bailey, Conisbee y Gaehtgens. The Age of Watteau..., p. 88.

18. CARPERNTER, Richard. The Dutch Sources of the Art of J.-H. Fragonard. Ph.D diss. Harvard University, 1955. BroOKner, Anita. Greuze: The Rise and Fall of an $18^{\text {th }}$ Century Phenomenon. Londres: Elek, 1972. Snoep-Reitsma, Ella. Chardin and the Bourgeois Ideals of his Time. Nederland Kunstbistorisch Jaarbock, 1973, 24, pp. 147-243. BANKS, Oliver T. Watteau and the North: Studies in the Dutch and Flemish Baroque influence on French Rococo Painting. Nueva York: Garland Publishing, 1977. Oursel, Hervé (com.). Au temps de Watteau, Fragonard e Chardin: Les Pays-Bas et les peintres français du XVIII siècle. Lille, 1985. ATWATER, Vivien Lee. A Catalogue and Analysis of Eighteenth-Century French Prints after Netherlandish Baroque Painting. Ph.D. diss. Washington University, 1988. CornelIs, Bart. "Arnold Houbraken's Groute Schoubourgh and the Canon of Seventeenth-Century Dutch Painting". 
el paradigma del realismo burgués, de la pintura de género y de lo cotidiano, no es de extrañár que la pintura francesa dieciochesca (reducida por ambas exposiciones a la pintura de lo cotidiano) no solo compartiera el mismo principio explicativo que la pintura holandesa: la emergencia de la burguesía, sino que para entender sus transformaciones se debía acudir a aquella supuesta influencia: "depictions of Dutch bourgeois reality [...] giving artistic expression to the viewer's own hopes for social change. In this sense, it is sometimes suggested that Dutch painting spoke to the growing self-assurance of the bourgeoisie in Paris during the eighteenth century» ${ }^{19}$. Ambas exposiciones dejaban de lado la poética clásica como el elemento principal que permitiría comprender las transformaciones en la pintura francesa del siglo XVIII, para subrayar, por el contrario, lo popular y la influencia del arte holandés ${ }^{20}$.

A continuación realizaremos un breve recorrido en torno a las transformaciones producidas en el término pintura de género desde finales del siglo XVIII. En primer lugar, se analizará cómo las discusiones alrededor de la jerarquía de los géneros durante la Revolución francesa (en un contexto donde se cuestiona la Academia) van a crear nuevos sentidos adheridos al término género, que comenzará a identificarse con la pintura holandesa, lo popular, etc. A medida que las transformaciones sociales y políticas del siglo XIX transforman los debates poetológicos del siglo XVIII, asociándose la pintura de género a las reivindicaciones políticas y a los nuevos debates estéticos. En segundo lugar, se analizará cómo estos debates serán retomados por la tradición marxista del siglo xx, incorporándose a las discusiones sobre el realismo de los años 30. Unas ideas que emergen de nuevo en los años 70, donde las interpretaciones hegelianas de los escritos de 1844 del joven Marx -deudoras del mundo francés- favorecieron en el mundo anglosajón una comprensión cultural de las dinámicas históricas y sociales y, por tanto, una interpretación del género en términos de cotidianidad. En tercer lugar, se analizarán las principales aproximaciones sociales a la pintura francesa del siglo XVIII en el mundo angloamericano y cómo los debates políticos de la New Left y las nuevas corrientes historiográficas como los Cultural Studies o la New Art History, condicionaron su interpretación.

Simiolus, 1998, XXVI, 3, pp. 144-161. AlTES, Everhard Korthals. "The Eighteenth-Century Gentleman Dealer Willem Lormier and the International Dispersal of Seventeenth-Century Dutch Painting". Semiolus, 2000/2001, XXVIII, 4, pp. 251-311.

19. Gaehtgens, Thomas W. "Genre Painting in Eighteenth-Century Collections». En Bailey, Conisbee y Gaehtgens. The Age of Watteau..., p. 80.

20. Bailey, Colin B. "Surveying Genre in Eighteenth-Century French Painting». En Bailey, ConisBeE y Gaehtgens. The Age of Watteau..., p. 18. 


\section{DE LA JERARQUía DE LOS GÉNEROS AL GÉNERO SOCIAL}

Richard Rand y Colin B. Bailey intentaron encontrar en los textos del dieciocho una definición de pintura de género similar a la que proponían en sus respectivas exposiciones, aunque sin mucho éxito, pues en el siglo XVIII la pintura de género se inscribía dentro de los principios poetológicos del clasicismo (desarrollados en pintura por Félibien), los cuales no fueron discutidos hasta la Revolución. Momento en el que se describió la existencia de una supuesta jerarquía de los géneros dentro del pensamiento académico, la cual debía cuestionarse, surgiendo, así, las primeras voces que explícitamente defendieron la pintura de género frente a la hegemonía de la pintura de historia ${ }^{21}$. En un contexto político donde se estaba cuestionando también la hegemonía académica, la pintura de género se intentó adscribir a los valores revolucionarios ${ }^{22}$ y a los principios morales del pueblo (fuente de regeneración social), tomándose como referencia -en ocasiones- la pintura holandesa ${ }^{23}$.

Será en el año II de la Revolución (1794), cuando se producirán diversos acontecimientos importantes en relación al debate sobre la jerarquía de los géneros en la Société populaire et républicaine des arts; y, así, el 13 germinal (2 de abril de 1794), Jean Baptiste Pierre Lebrun señala: "Nous devons beaucoup à l'École Flamande et que ces Maîtres, outre la couleur, ont peint souvent des Tableaux utiles, en nous retragant les vertus des chaumières des familles simples ${ }^{24}$. Lebrun defendía no solo la pintura flamenca, sino la pintura de género en general, oponiéndose con ello a la defensa realizada por David sobre las posibilidades expresivas de la pintura de Historia ${ }^{25}$ :

Nous ne pouvons nos dissimuler que la plupart des grands traits de notre révolution, la fermeté, les vertus, le dévouement de nos frères, n'offreant, pour ainsi dire, à l'art que des tableaux de genre, si l'Artiste ne ce sauve pas par d'ingénieuses allégories, langage qu'on n'entend pas toujours acsément [...] Le genre frappe tous les yeux et tous les hommes ${ }^{26}$.

21. Olander, William. "French Painting and Politics in 1794: The Great Concours de l'an II». En Wintermute, Alain (com.). 1789: French Art During the Revolution. Nueva York: Colnaghi, 1989, pp. 29-45. Eliel, Carol S. "Genre Painting During the Revolution and the Goût Hollandais». En WinTERMUTE, 1789: French Art During the Revolution..., pp. 47-61. VAN DE SANDT, Udolpho. "Grandissima opera del pittore serà l'historia. Notes sur la hiérarchie des genres sous la Révolution». Revue de l'Art, 1989, 83, pp. 71-76. Whiteley, Jon. «Art, hiérarchie et Révolution française». En RoQuE. Majeur ou mineur..., pp. 67-77.

22. Pommier, Édouard. L'Art de la liberté. París: Gallimard, 1991, p. 196.

23. SPIETH, Darius. Revolutionary Paris and the Market for Netherlandish Art. Ámsterdam: Brill, 2017.

24. Détournelle, Athanase. Aux armes et aux arts! Peinture, sculpture, architecture, gravure. Journal de la Société républicaine des arts séant au Louvre. París, 1794, pp. 267-268.

25. DAVID, Jacques Louis. Rapport fait à la Convention National au nom du Comité d'Instruction Publique sur la nomination des cinquante membres du jury qui doit juger le concours des prix de peinture, sculpture et architecture, 25 brumaire, an II (15 de noviembre de 1793).

26. LeBrun, Jean Baptiste Pierre. Essai sur les moyens d'encourager la peinture, la sculpture, l'achitecture et la gravure. París, an III, p. 9. 
Adscrito a aquellos debates que cuestionaban la jerarquía de los géneros, Lebrun señalaba que el género representará la virtud de las cabañas y de las familias simples, considerándola la encargada de representar los grandes acontecimientos revolucionarios ${ }^{27}$ :

Tous les Artistes quelconques, Peintre d'histoire, pourront aussí concourir en traitant les plus beaux sujets qu'offre notre révolution [...] Les Peintres appelés de genre, ne sont point indignes de l'attention de la Nation. Genre n'est qu'un mot, et c'est beaucoup faine que de rendre la nature avec choix et fidélité [...] Nous ne pouvons nous dissimuler que la plûpart des grands traits de notre révolution, la fermeté, les vertus le dévouement de nos frères, n'offrent, pour ainsi dire, à l'art que des tableaux de genre ${ }^{28}$.

A pesar de esta defensa, la pintura de historia seguirá ocupando un lugar preponderante durante las primeras décadas del siglo XIX, en un siglo obsesionado por la Historia: "Tout prend aujourd'hui la forme de l'histoire»" Esta se vio beneficiada, además, por los triunfos militares y por el culto a la personalidad de Napoleón, favoreciéndose la pintura de batallas ${ }^{30}$; así como por el predominio de los alumnos de David que ayudaron a mantener su lugar hegemónico. No obstante, la jerarquía de los géneros irá perdiendo paulatinamente su preponderancia, en paralelo a la transformación de la noción de Historia como magistra vitae y al declive de los alumnos de David ${ }^{31}$. Un proceso que coincide con el abandono de la pintura de historia en favor de lo que se ha definido como género histórico o anecdótico ${ }^{32}$, y que Delécluze achacó al fomento de la pintura de batallas y al estilo troubadour ${ }^{33}$ :

27. Bordes, Philippe. Représenter la Révolution. Les Dix-Août de François Gérard et de Jacques Bertaux. Lyon: Fage, 2010.

28. LEBRUn. Essai sur les moyens d'encourager.., p. 7 y p. 9.

29. Chateaubriand, François René de. Études ou discours historiques sur la chute de l'Empire romain. París: Eugène et Victor Penaud Frères, 1831, p. 46.

30. SIEGFRIED, Susan L. "L'Iconographie militariste sous la Révolution et l'Empire». En VovelLE, Michel (ed.). Les Images de la Révolution française. París: Publications de la Sorbonne, 1988, pp. 93-96.

31. Allard, Sébastien y Chaudonneret, Marie-Claude. Le Suicide de Gros. Les peintres de l'Empire et la génération romantique. París: Gourcuff Gradenigo, 2010.

32. Wright, Beth S. Painting and History During the French Restoration: Abandoned by the Past. Cambridge: Cambridge University Press, 1993. CHAUdOnNERET, Marie-Claude. "Du "genre anecdotique" au "genre historique": Une autre peinture d'histoire». En Julia, Isabelle y LaCAmbre, Jean (coms.). Les années romantiques: la peinture française de 1815 à 1850. París: RMN, 1995, pp. 76-85. AMBrosinI, Lynne. "Genre Painting under the Restoration and July Monarchy: The Critics Confront Popular Art». Gazette des Beaux-Art, 1995, 1512, 125, pp. 41-52. BANN, Stephen. "Question of Genre in Early Ninteenth-Century French Painting». New Literary History, 2003, 34, 3, pp. 501-511. Duro, Paul. "Giving up on History? Challenges to the Hierarchy of the Genres in Early Ninteenth-Century France». Art History, 2005, 28, 5, pp. 689-711. BAnN, Stephen. "Au fil de l'histoire: généalogie des genres "anecdotique" et "historique"». En Bann, Stephen; Paccoud, Stéphane y Briat-Philippe, Magali (coms.). L'invention du passé. II. Histoires de cour et d'épée en Europe, 1802-1850. París: Hazan, 2014, pp. 12-25.

33. DelÉcluze, Jean-Étienne. "Sur l'exposition des ouvrages de peinture, sculpture...». Le Lycée française, 1819, 1, pp. 269-278. 
Une des choses très remarquables dans l'exposition de cette année, Sire, c'est la multiplicité de tableaux d'un genre qui appartient particulièrement à l'école français, par sa délicatesse et son instruction. C'est l'histoire anecdotable ou la représentation de personnages dont la vie historique fait désiner de s'approcher, pour ainsi dire, d'eux et de connaître leur vie privée, genre qu'on pourrait appeler la comédie noble ou le drame de la peinture ${ }^{34}$.

La extensión del género a lo largo del siglo XIX transformará la pintura de historia y, de este modo: "Les peintres d'histoire même ne craignent pas d'abaisser la majesté de leurs pinceaux jusqu'à des scènes familières » ${ }^{35}$. Esto favorecerá una confusión terminológica que se acentúa a partir de la Restauración, cuando los diversos grupos políticos parecen ir tomando partido por una u otra. Un artículo anónimo de 1821, del entorno del periódico liberal Miroir des spectacles, muestra así su oposición a la aristocracia de los géneros, identificado la jerarquía de los géneros con los estamentos sociales, considerándolos como «une des absurdités les plus grandes qu'on ait pu soutenir en matèrie d'arts", ya que "Toute discussion sur la prééminence à accorder à l'un d'eux, au préjudice des autres, est une puérilité peu digne d'occuper les nons sprits", concluyendo que "toute acception de genre est une erreur ${ }^{36}$. Entre los críticos conservadores aparece de forma constante, por contra, la defensa de la jerarquía de los géneros y, de este modo, en 1824 en el periódico monárquico La Gazette de France se señala: "Le peintre d'histoire en grand l'emportera toujours sur le peintre de genre, par l'importance des travaux, des études, et des difficultés innombrables de l'exécution ${ }^{37}$. Todo ello generó una creciente confusión a la hora de definir la pintura de género y de historia, como reconocía el crítico Adolphe Thiers: "Mais ce titre de genre, objet de discussions très-vives, n'a pas encore été bien déterminé [...] Peu importe, au surplus, la classe à laquelle appartienne un tableau; l'essentiel est qu'il soit bon ${ }^{38}$. Más adelante el mismo autor reconoce cómo los artistas están abandonando la pintura de historia por una pintura de costumbres y verdadera: "On se plaint beaucoup aujourd'hui de cette désertion générale des artistes qui abandonnent l'histoire pour peindre le genre. J'avoue que je m'en alarmerais peu [...] j’aime aussi ces jolies peintures

34. Vivant-Denon, Dominique. Rapport à l'Empereur, 11 novembre, 1810. Arch. Nat., AF. IV 1050, dossier 6. Citado en Chaudonneret, Marie-Claude. "Du "genre anecdotique" au "genre historique"...", p. 76.

35. MÉly-Janin, Marie. "Salon de 1824». La Quotidienne, 19 nov., 1824. Citado por AmbrosinI. "Genre Painting under...", p. 44

36. Anonyme. "La Bataille de Jemmapes, par M. Horace Veernet». Le Miroir des spectacles, des lettres, des modes et des arts, 7 marzo de 1821.

37. Chauvin. Salon de mil huit cent ving-quatre. París: Pillet, 1825, p. 99.

38. ThiERs, Adolphe. "Salon de 1822". En THIERs, Adolphe. Collection des articles insérés au Contitutionnel. París: Maradan, 1822, p. 112. 
de mours rendues avec vérité et avec esprit ${ }^{39}$; mostrando una clara defensa del género en su crítica al Salón de 1824:

Lorsque, des rangs élevés où sont suspendues au Salon les grandes toiles, on porte les yeux sur cette foule de petits tableaux placés à hauteur d'appui, on éprouve une satisfaction véritable, et qui console de l'incertitude, de l'anxiété où nous a laissés le plus souvent la peinture d'histoire [...] Nous ne disons pas que nous devions renoncer à l'histoire, car de belles et immortelles productions seraient là pour nous contredire et pour condamner une pareille renonciation; mais il est certain que nous n'avons tout notre génie qu'en peignant le genre: alors, nous sommes nous-mêmes; alors, nous entrons en possession de nos qualités, et nous sommes en situation pour les déployer toutes ${ }^{40}$.

Esta defensa del género se acentuará, sobre todo, en los umbrales de la revolución de 1830: "Notre nouvelle école est une république où le petit peuple est plus instruit et plus avancé que les grands seigneurs» ${ }^{41}$; convirtiéndose en un lugar de confrontación política ${ }^{42}$ entre los defensores y detractores de la restauración borbónica. Entre las diversas causas que van a determinar esta transformación de los valores estéticos heredados del siglo XVIII ocuparán un lugar destacado, en primer lugar, el auge del Salón y de un mercado de arte privado creciente en detrimento del Estado (a pesar de los esfuerzos de este) ${ }^{43}$; y, en segundo lugar, la eclosión de la crítica artística, que condicionará cada vez más la producción artística, como reflejaría la trayectoria profesional de Courbet $^{44}$. Bajo el Imperio napoleónico y ante la férrea censura de este, los ataques al régimen se habían ocultado tras la crítica artística, determinando una politización de esta ${ }^{45}$ que se acentuará por la proliferación de los periódicos bajo la Restauración y la Monarquía de Julio ${ }^{46}$; lo que muestra el lugar preponderante ocupado por la crítica en la sociedad: «Depuis

39. Ibid., p. 121.

40. ThIERS, Adolphe. «Salon de 1824». Le Globe, 16 oct., 1824, p. 63.

41. VITET, Ludovic. "Salon de 1827». Le Globe, 1828, 6, p. 181.

42. Chaudonneret, Marie-Claude. "Art et Politique». En Bann, Paccoud y Briat-Philippe. L'invention du passé..., pp. 26-33.

43. Marrinan, Michael. Painting Politics for Louis-Philippe. Art and Ideology in Oréanist France, 1830-1848. New Haven: Yale University Press, 1988. CHAUdONNERET, Marie-Claude. L'État Eles Artistes. De la Restauration à la monarchie de Juillet (1815-1833). París: Flammarion, 1999.

44. CHu, Petra Ten-Doesschate. The Most Arrogant Man in France. Gustave Courbet and the Ninteenth-Century Media Culture. Nueva Jersey: Princeton University Press, 2007.

45. CABAnIs, André. La presse sous le Consulat et l'Empire (1799-1814). París: Société des Études Robespierristes, 1975. SIEGFRIED, Susan L. "The politicisation of art criticism in the post-revolutionary press». En Orwicz, M. (ed.). Art Criticism and its Institutions in 19th Century in France. Manchester: Manchester University Press, 1994, pp. 9-28. PERRET, Irène. La critique d'art sous le Consulat et le première Empire (1799-1815). Thèse sous la direction de Bruno Foucart. Université Paris IV, 2008.

46. MCWILliam, Neil. "Opinions professionneles: critique d'art et économie de la culture sous la Monarchie de Juillet». Romantisme, 1991, 71, pp. 19-30. McWilliam, Neil. "Presse, journalistes et critiques d'art à Paris de 1849 à 1860”. Quarante-buit/Quatorze, 1993, 5, pp. 53-62. 
ce temps, les choses ont repris leur cour; la critique est revenue jouer son véritable rôle; elle a retourné la société dans tous les sens» ${ }^{47}$. Nos encontramos, pues, ante una producción periodística -donde se inscribe la crítica artística- de una gran variedad y complejidad en sus intereses políticos y estéticos ${ }^{48}$, no siempre fácil de desentrañar y donde la jerarquía de los géneros ${ }^{49}$ será redefinida entre aquellos que están a favor o en contra de culminar las transformaciones inconclusas de la Revolución.

Este desarrollo de la prensa puso de manifiesto la emergencia de una nueva experiencia temporal centrada en el presente. Un cambio en el régimen de historicidad ${ }^{50}$-alumbrado por la Revolución- que rompía con la noción de historia anterior y que favoreció una nueva experiencia temporal que a través de la crítica artística animó igualmente al arte a centrarse en su presente. El auge de una pintura de género que progresivamente mira al mundo circundante mostraba cómo la realidad se impuso como un nuevo material sobre el cual el arte buscó repensarse ${ }^{51}$ : «Malheure à celui qui étudie dans l'antique [...] Pour s'y trop plonger, il perd la mémoire du présent; il abdique la valeur et les privilèges fournis par la circonstance ${ }^{52}$. Este impulso hacia lo real, que Auerbach habia descrito en distintos momentos de la historia occidental ${ }^{53}$, parecía adquirir en este siglo XIX un lugar preponderante. Esto puede comprenderse, asimismo, como una respuesta a la sospecha que sobre la imagen del mundo se había cernido desde las Críticas kantianas y que había situado la cuestión de la falsedad del mundo en un lugar prioritario en todos los ámbitos de pensamiento. El impulso antropológico consecuente que animará el pensamiento del siglo XIX ${ }^{54}$ consideró, sin embargo, que tras aquella falsedad era posible acceder a una verdad más fundamental y original, convirtiéndose la realidad en el camino y en el material sobre el que trabajarán tanto las ciencias sociales como las artes, que buscarán la Verdad a través de lo real: «Le génie poétique est mort, mais le génie du soupçon est venu au monde.

47. AnONyme. "Des avantages de la critique». Le Globe, 2 de mayo de 1826, t. III, n. ${ }^{\circ}$ 56, p. 300.

48. Kalifa, Dominique; RÉGnier, Philippe y ThÉREnTy, Marie-Ève (dirs.). La civilisation du journal: Histoire culturelle et littéraire de la presse française au XIXe siècle. París: Nouveau Monde Éditions, 2011.

49. Vergnette, François de. "Débats sur la hiérarchie des genres et sur la définition dela peinture d'histoire sous la Restauration et la Monarchie de Juillet». En LachEnAL, Lucie y MÉnEux, Catherine (eds.). La critique d'art de la Révolution à la Monarchie de Juillet. París: L'HiCSA, 2013, pp. 291-212.

50. Koselleck, Reinhart. Le Futur Passé. Contribution à la sémantique des temps historiques. París: EHESS, 1990. HaRTOG, François. Régimes d'historicité. Presentism et expériences du temps. París: Seuil, 2012.

51. Dufour, Philippe. Le Réalisme: de Balzac à Proust. París: PUF, 1998.

52. Baudelaire. Euvres Complètes. París: Gallimard, 1976, t. II, p. 696.

53. AUERBACH, Erich. Mimesis. La representación de la realidad en la literatura occidental. México: FCE, 2006.

54. Foucault, Michel. Las palabras y las cosas. México: Siglo XXI. 
Je suis profondement convaincu que le seul antidote qui puisse faire oublier au lecteur les éternels Je que l'auteur va écrire, c'est une parfaite sincérité, ${ }^{55}$.

En paralelo a este interés por el mundo circundante, diversos críticos defendieron una pintura verdadera, que algunos identificaron con la pintura de género y otros describieron como realismo, tal y como hará Laviron en 183156, Planche en $1833^{57}$ o Charles Blanc y Thoré en los años 40. Sin embargo, esta realidad será comprendida de muy diferente forma dependiendo de cada artista, mostrando que el realismo era una cuestión de convención estética, una especie de táctica empleada por diversos artistas como estrategia para diferenciarse de otras generaciones o propuestas estéticas ${ }^{58}$. Según señala el Trésor de la langue française, el término realismo había surgido por vez primera en 1826, en un artículo del Mercure français $d u X_{X I}{ }^{e}$ siècle ${ }^{59}$, siendo frecuente en las primeras décadas del siglo XIX, lo cual revela un impulso temprano del siglo por indagar sobre la realidad circundante. No obstante, será durante el Segundo Imperio, caracterizado, por un lado, por la crisis económica que atrae a grandes masas campesinas a la ciudad, aumentanto conflictividad social; y, por otro lado, por un fenómeno industrializador que busca mejorar las vías de comunicaciones, lo que permitía tomar contacto con la realidad francesa del campo y sus diferentes regiones; cuando en la crítica se observa el fomento de una pintura de género vinculada a lo cotidiano, a las diferencias regionales, a sus diversos paisajes, etc. ${ }^{60}$.

La paysannerie rêveuse et sentimentale est bien plu un fruit de notre siècle que le paysage. Autrefois, on ne peignait les villageois que pour en tirer des effets comiques; nous leur demandons aujourd'hui l'expression raffinée des passions [...] C'est seulement de notre temps et depuis peu d'annés qu'on a véritablement transporté le paysan dans le domaine de l'art [...] nous avons pu voir le village tout entier envahir nos expositions ${ }^{61}$.

55. STEndHal. Souvenirs d'égotisme. 1832. París: Le Divan, 1927, p. 6.

56. BÉNÉDite, Léonce. Notre art-nos maîtres. París: Flammarion, 1923, p. 91.

57. Grate, Pontus. Deux critiques d'art de l'époque romantique: Gustave Planche et Théophile Thoré. Estocolmo: Almqvist and Wiksell, 1959, p. 99.

58. JAKOBSON, Roman. "Sobre el realismo artístico. 1921». En Todorov, Tzvetan (ed.). Teoría de la literatura de los formalistas rusos. México: Siglo XXI, 2007.

59. "Cette doctrine littéraire qui gagne tous les jours du terrain et qui conduirait à une fidèle imitation, non pas des chefs-d'œuvre de l'art, mais des originaux que nous offre la nature, pourrait très bien s'appeler le réalisme: ce serait suivant quelques apparences, la littérature dominante du XIXe siècle, la littérature du vrai» Mercure français du XIX siècle. París: Au bureau du Mercure, 1826, vol. 13, p. 6.

60. VotTero, Michaël. La peinture de genre en France, aprés 1850. Rennes: PUR, 2012, p. 32. Herbert, Robert L. "City vs. Country: The Rural Image in French Painting from Millet to Gaugin". Artforum, 1970, VIII, pp. 44-55. DOUCE DE LA SAlLE, Sylvie. "Les representations de paysans, 1852-1870". En LE NouËNE, Patrick (com.). Exigences de réalisme dans la peinture française entre 1830 et 1870. Chartres: Musée des Beaux-Arts, 1983, pp. 134-158.

61. Calonne, Alexis de. "La Peinture Contemporaine à l'exposition de 1861". Revue Contemporaine, 1861, mai-jun, pp. 341-342. 
El Segundo Imperio favoreció asimismo, como reacción a la crítica radical de la Segunda República, una pintura social, apoyando en los años 50 una crítica literaria donde adquirió un lugar predominante el término realismo, que se convirtió en un espacio de conflicto estético y político, determinando la dificultad actual a la hora de poderlo definir ${ }^{62}$. En 1856 Duranty funda su revista Réalisme. En 1855 Gustav Courbet titula su exibición personal, realizada a propósito de la Exposición Universal, como Du Réalisme: "Le titre de réaliste m'a éte imposé comme on a imposé aux hommes de 1830 le titre de romantique» ${ }^{63}$. A partir de 1855 aparecerán igualmente diversas obras que tratarán esta cuestión, como la obra de Max Buchon Le Réalisme, de 1856, o de Champfleury Le Réalisme, de $1857^{64}$. No obstante, y pese a los deseos por vincular el realismo a ciertos posicionamientos políticos de carácter social, observamos en la definición dada por Duranty ${ }^{65}$ que por verdad o realismo se expresaba un interés creciente por el mundo contemporáneo; renegando del término autores como Flaubert, Courbet o el propio Baudelaire, quien lo consideraba como una farsa de su amigo Champfleury.

Desde la Monarquía de Julio asistimos, pues, a la proliferación de una pintura de género cada vez más diversa, lo que animará a los críticos a intentar encontrar una definición:

Tout ce qui n'est ni tableau d'histoire, ni paysage, ni marine, est du genre. [...] il ne doit jamais dépasser les limites d'un tableau de chevalet. Le genre se détermine encore d'après l'importance des sujets: l'anecdote, les mœurs rustiques, les scènes familières, sont principalment de son domaine ${ }^{66}$.

A medida que la confrontación política se agudiza, la oposición entre género e historia también se acentuará. A raíz de lo cual una parte de la crítica socialista, heredera de ciertos posicionamientos de Saint-Simon ${ }^{67}$, reivindicará una pintura social que mire al drama cotidiano y a lo humano desde la cual regenerar la sociedad $^{68}$, animando así a superar el individualismo romántico de l'art pour l'art. El

62. Chamarat, Gabrielle y Dufief, Pierre-Jean (eds.). Le realisme et ses paradoxes (1850-1900). París: Garnier, 2014.

63. Courbet, Gustave. "Prólogo al catálogo de su exposición”. En Exhibition et vente de 40 tableaux et 4 dessins de M. Gustave Courbet, en el Pavillon de l'Avenue Montaigne. París, 1855.

64. Troubat, Jules. Une amitié à la D'Arthez, Chamfleury, Courbet, Max Bouchon... París: Lucien Duc, 1900.

65. Duranty, Edmond. «Realisme». Realisme, 15 de noviembre de 1856, pp. 1-2.

66. BARBIER, Alexandre. Salon de 1836. París: Renouard, 1836, p. 97.

67. McWilliam, Neil. Dream of Happiness. Social Art and the French Left, 1830-1850. Nueva Jersey: Princeton University Press, 1993.

68. HunT, Herbert. Le socialisme et le romantisme en France. Étude de la presse socialiste de 1830 à 1848. Oxford: Clarendon, 1935. 
crítico radical Théophile Thoré ${ }^{69}$ defenderá un nuevo arte natural y humano que progresivamente identificará con lo cotidiano.

Mais aujourd'hui que la société se régénère par de nouvelles croyances, l'art peut se concevoir une mission, sa mission sainte et éternelle de diriger les hommes dans les voies de la Provindence, autrement de leur amélioration et de leur perfectionnement [...] la plupart ont encore une singulière prédilection pour la peinture historique [...] et n'avons-nous pas des drames assez vivans au milieu de nous? [...] Je le répète, faire de l'actualité, descendre au cour de l'époque, rendre aux beaux-arts leur caractère social, voilà l'évolution à consommer ${ }^{70}$.

El pueblo se convertía así en el tema principal de las representaciones artísticas y de la crítica: "Ce qui domine partout c'est l'amour et l'intelligence du peuple ${ }^{71}$; así como de las diversas facciones políticas ${ }^{72}$. Todo ello muestra cómo el principal objetivo del arte era la representación de la vida contemporánea, como señala el periódico republicano La Tribune: «Mais il faut... que toute pensée d'art soit désormais inspirée du présent, de ses passions, de ses misères, que toute faintaisie d'art ait son but, sa volonté, son action dans ce monde actif ${ }^{73}$. Esta reivindicación de un arte social ejemplar, la encontraremos también entre los críticos afines a Thoré de la revista republicana nonata La Démocratie:

Ce que nous demandons, ce n'est pas de la peinture utile, de la peinture qui servirait comme le bronze d'un bourgeois, ce que nous voulons, c'est qu'on utilise les arts, c'est qu'on leur fasse retracer de belles actions pour les répandre en profusion sous les yeux des masses; c'est enfin qu'on appliquer leur puissans effet à l'amélioration de la chose sociale. Tout ajourd'hui doit avoir une doctrine formulée, un but social ${ }^{74}$.

La obra de Thoré constituye así un punto de inflexión dentro de esta definición moderna de la pintura de género. Aunque su defensa de un nuevo arte no se construirá tanto sobre la representación de las miserias del pueblo, sino sobre la espiritualidad de la naturaleza, donde se encuentra para él la ejemplaridad moral que antes ocupaba la Historia ${ }^{75}$. Una espiritualidad que también reside en lo humano y en el pueblo, tal y como ejemplificaría para él la pintura holandesa: «Il n’y

69. Jowell, Frances Suzman. Thoré-Bürger and the Art of the Past. Nueva York: Garland Publishing, 1977.

70. ThORÉ, Théophile. «L'art social et progressif». L'Artiste, $1834,1^{\text {st }}$ ser., n. ${ }^{\circ}$ 7, p. 41.

71. HauréAu, Barthélémy. La Tribune politique et littéraire, 1833. Citado en LE NouËNE, Patrick. "Representations du peuple dans les Tableaux d'Histoire exposes au Salons entre 1831 et 1848". En LE NOUËNE, Patrick. Exigences du réalisme..., pp. 27-28.

72. Pessin, Alain. Le mythe du peuple et la société française du XIX siècle. París: PUF, 1992.

73. La Tribune politique et littéraire, 15 de marzo de 1834.

74. Schoelcher, Victor. "Salon de 1835 (deuxième article)». Revue de Paris, 1835, vol. XV, p. 45.

75. GRATE. Deux critiques d'art..., pp. 182-184. 
a plus qu'une race et qu'un peuple, il'y a plus qu'une religion et qu'un symbole: l'humanité» ${ }^{76}$.

Entre 1815 y 1848 , en paralelo al desarrollo de la pintura de género y a las reivindicaciones de un arte social, asistiremos también a un renovado interés por la pintura holandesa del siglo XVII ${ }^{77}$, favorecido por el desarrollo de la novela; lo que determinará la asociación entre las búsquedas estéticas de la novela, del realismo y de la pintura holandesa ${ }^{78}$. Esto determinará que nuestra imagen de la pintura holandesa actual sea en gran parte un producto del siglo XIX ${ }^{79}$. Momento en que se convirtió en una categoría más de la crítica artística a través de la cual la novela y la pintura de género fueron analizadas. La pintura holandesa del siglo XVII se connotó, pues, con unos rasgos ajenos a su época, proyectándose sobre ella las ideas y anhelos políticos del siglo XIX ${ }^{80}$, describiéndose la Holanda del siglo XVII y la Francia de los años 30 y 40 del siglo XIX como la consecuencia de una misma necesidad social, esto es, de una clase burguesa emergente que debía transformar la sociedad a partir de su independencia, logrando construir una república libre y democrática, abandonando el yugo absolutista: "Il nous paraît, au contraire que les mours actuelles de la France se rapprochet plus de la bourgeoisie hollandaise du dix-septième siècle que d'aucune autre analogie historique» ${ }^{81}$. El propio Thoré ve en los pequeños géneros de la pintura holandesa la encarnación de sus aspiraciones políticas en los umbrales de la revolución del 48: la democratización de la sociedad y definición de un arte como centro de la educación democrática del pueblo a través de la belleza que se encuentra en lo humano.

Où trouver, chez n'importe quel peuple, une histoire plus conscieuse, plus naïve et plus spirituelle, plus vivante, que cette histoire peinte des mours et des actions? C'est la peinture qui a écrit l'histoire des Pays-Bas, et même une certaine histoire de l'humanités2.

76. ThORÉ, Théophile. "Nouvelles tendences de l'art». En Introduction, Salons de Théophile Thoré. París, 1868, pp. XVII-XVIII.

77. CHu, Petra Ten-Doesschate. French Realisme and the Dutch Masters, the Influence of Dutch Seveentheenth-Century Painting on the Development of French Painting between 1830 and 1890. Utrech: Haentjens Dekker \& Gumbert, 1974. WeIsBERG, Gabriel (com.). The Realistic Tradition, French Painting and Drawing, 1830-1900. Cleveland: The Cleveland Museum of Art, 1980.

78. YeAzeLl, Bernard. Art of the Everiday. Dutch Painting and the Realistic Novel. Nueva Yersey: Princeton University Press, 2008.

79. Grijzenhout, Frans y VAn Veen, Henk. «Introducción». En Grijzenhout, Frans y VAn VeEn, Henk (eds.). The Golden Age of Dutch Painting in Historical Perspective. Cambridge: Cambridge University Press, 1999, p. 7.

80. AMBRosini. "Genre Painting under...", p. 45.

81. ROYER, Alphonse. «Salon de 1840: genre». Le Siècle, 4 de abril de 1840.

82. Thoré, Théophile. «Nouvelles tendences de l'art». En THORÉ, Théophile. Introduction, Salons de Théophile Thoré. París: 1868, p. XXX. 
Para Thoré la pintura de historia era incapaz de captar lo humano, contraponiendo en la propia historia de la pintura francesa el grand genre a la petit école de Watteau, Chardin, Greuze, Boucher o Fragonard; a los cuales considera como la única y auténtica escuela francesa ${ }^{83}$ (tal y como se manifestará en las exposiciones arriba estudiadas). A partir de la filosofía de la historia ${ }^{84}$, Thoré construyó un nuevo relato sobre la historia del $\operatorname{arte}^{85}$ (tal y como estaban realizando también Charles Blanc o Théophile Silvestre), donde era el género el que daba sentido al relato histórico. Pero también se definía el género como un lugar de toma de conciencia histórica sobre lo humano: "L'humanité a pris conscience d'elle-même le long du temps, et conquis en quelque sorte la possesion de son immortalité „ ${ }^{86}$. Será en su Musée de la Hollande donde Thoré culminará todas estas ideas que ponían en paralelo la pintura holandesa y la pintura de lo humano como fuente de renovación para el arte contemporáneo, ocupando la realidad un lugar prioritario: "Devant la réalité s'évanouiront bientôt, forcément, les abstractions, conventionnelles, et un idéal tout naturel se dégagera des saines impressions ressenties par les artistes ${ }^{87}$. Con él la pintura de género se redefinirá en un sentido social que marcará la tradición socialista posterior ${ }^{88}$.

Si el Salón de 1868 había consagrado la pintura de género otorgándole el honor del primer premio, sin embargo, con la Tercera República el Estado intentará devolver a la pintura de historia su lugar preeminente pasado. Pero esta se inscribirá dentro de una comprensión social del arte que de facto reflejaba el triunfo de los postulados estéticos adscritos a la pintura de género y, de este modo, si bien continúa pensándose el género en contraposición a la pintura de historia, sin embargo, durante la Tercera República la pintura de género se identificó definitivamente con la vida real y con lo humano:

L'expression peinture de genre a été imaginée par la critique moderne, pour désigner les compositions dont les sujets sont empruntés à la réalité et copiés, pour ainsi dire sur le vif, par opposition à celles qui expriment, d'une façon idéale, des scènes historiques religieuses ou de pure fantaisie, et qui constituent la "peinture d'histoire». [...] Le mot genre n'a pas aujourd'hui une acception aussi large que cette qu'il avait autrefois. Il ne s'emploie plus que pour désigner les peintures de

83. MCCOuBrey, John W. "The revival of Chardin in French Still-Life Painting, 1850-1870". Art Bulletin, 1964, vol. XLVI, 1964, pp. 39-53. WeIsBERG, Gabriel y TALBOT, William S. (coms.). Chardin and the Still-Life. Tradition in France. Cleveland: Cleveland Museum of Art, 1979.

84. THORÉ, Théophile. "Salon de 1838». Revue de Paris: nouvelle série, 1838, vol. LII, p. 39.

85. KoHLE, Hubertus. "Spontanéité et reconstruction. Du rôle de l'organisation et des compétences de la critique d'art par rapport à la théorie de l'art et à l'histoire de l'art». En KoHLE, Hubertus. Arts et société. BoD, 2009, pp. 129-159. p. 78

86. ThORÉ, Théophile. "Des tedances de l'art au XIX siècle». Revue Universelle des Arts, 1855, 1,

87. ThOré, Théophile. "Salon de 1864». En ThORÉ, Salon de W-Bürger..., p. 91, t. II.

88. Rubin, James H. Realism and Social Vision in Courbet and Preudhon. Nueva Yersey: Princeton U. P., 1980 
mœurs familières, les scènes de l'existence rurale, les tableaux d'intérieur, la représentation des usages, des coutumes, des fêtes, des cérémonies, des travaux et des divertissements populaires; il s'applique, en un mot, à toute œuvre qui exprime un côté quelconque de la vie réelle, élégant ou misérable. Les paysages, les portraits, la nature morte, les fleurs, qu'on rangeait jadis dans le genre, forment aujourd'hui des catégories distinctes ${ }^{89}$.

Para el Grand Larousse fue en el siglo XVIII, con Greuze y Chardin, cuando el género alcanzó su más alta cota de expresión, mostrando, con ello, el profundo proceso de reelectura que del siglo XVIII se está haciendo en estos instantes: "La France possède, depuis plus d'un siècle, la suprématie dans la peinture de genre; aucune nation artiste, sans excepter la Hollande, n'a déployé dans la représentation des scènes de la vie domestique autant de finesse, de sentiment, de grâce, de vérité, de verve et d'esprit» ${ }^{90}$. El arte moderno -como señaló Huysmans en sus Salones de 1879 y 1880 - se centraba cada vez más en los acontecimientos de la vida cotidiana, dentro de un contexto político donde se defiende la necesidad de un arte social ${ }^{91}$, comprometido con la realidad social y que ayudase a su transformación; lo que favorecerá la proliferación de nuevos relatos sociales de la Historia del Arte, donde el pueblo y el realismo social se convierten en los dinamizadores de ese relato histórico:

La liste est longue de ceux qui cédèrent et constituèrent la peinture officielle française. Mais plus grand encore est le nombre de ceux qui ne cédèrent pas. En peignant l'immortelle Barricade, Delacroix a dressé contre la bourgeoisie de la Restauration le poème farouche de la Révolution républicaine. En fundant le réalisme pictural, Courbet, qui fut communard, représenta le second âge de ce mouvement protestataire. Et Manet incarna le troisième, en créant le modernisme. Parallèlement Daumier, génie libertaire aux sombres révoltes, stigmatisa la bourgeoisie dans ses dessins, et Millet osa peindre et imposer l'homme de la glèbe, la paysanne, en face de l'idéal académique, tandis que Courbet et Manet introduisaient dans leur art l'ouvrier et les types de la rue, comme jandis les Hollandais, les Falmands l'avaient osé, et comme l'avaient aussi osé nos sculpteurs gothiques et nos peintres du quatorzième et du quinzième siècle, interrompus par la Renaissance [...] L'école française reprit sa vraie direction, derivée par les ordonnances royales, celle du réalisme pittoresque [...] De Daumier, de Courbet, de Millet et de Manet date définitivement un gran fait: l'introduction de l'ouvrier, du paysan, de la femme du peuple, dans la peinture, l'acceptation de leurs vêtements et de leurs outils dans l'esthétique d'où ils

89. LAROUSSE, Pierre (ed.). "Genre». En Grand Larousse Universel du XIX siècle. París: AGDU, 1866-1877, t. 8, p. 1170.

90. LAROUSSE. "Genre...", p. 1170.

91. MARX, Roger. L'Art Social. Manifeste artistique et social. París: Eugène Fasquelle, 1913. MÉneux, Catherine (ed.). Regards de critiques d'art. Autour de Roger Marx (1859-1913). Rennes: PUR, 2009. 
avaient été bannis jusqu'alors. L'œuvre admirable de ces hommes a en pour complément une œuvre sociale, une glorification du travail dans ses humbles créatures ${ }^{92}$.

\section{LA HISTORIA SOCIAL DEL ARTE}

El auge de las ideas socialistas desde finales del siglo XIX favoreció el deseo de integrar el arte en la lucha ideológica ${ }^{93}$, por parte de muy diferentes grupos de izquierda que construirán la idea de un arte social ${ }^{94}$, abandonando aquellas corrientes adscritas a la erudición del conocedor. Asimismo, el proceso industrializador y el crecimiento del consumo -que estaba transformando la cultura a finales de siglofavoreció una mirada literaria y sociológica centrada en la ciudad y en la alienación de la vida moderna, como leemos en Simmel o en Benjamin, quienes retomando el impulso de autores como Baudelaire pondrán el acento sobre la cuestión de lo cotidiano $^{95}$. Un término que se convierte en un lugar común en la filosofía del momento, por ejemplo, en Heidegger o en Lukács.

Será la generación posterior a Marx y Engels quien reescribió y dio forma al corpus marxista, ante los desafíos a los que se enfrentaban los partidos de clase. Buscaron ofrecer una visión de conjunto, lo más coherente posible, frente a una cultura comprendida hasta ahora como un conjunto de valores de la clase dominante, como leemos en Plekhanov ${ }^{96}$ o Mehring ${ }^{97}$. En estos instantes se planteará también la existencia de un supuesto pensamiento artístico en Marx ${ }^{98}$, aunque ni Marx ni Engels concedieron excesiva atención a estas cuestiones ${ }^{99}$. No obstante, esta interpretación marxista ${ }^{100}$ del arte no fue más que una respuesta entre otras, entre quienes buscaban pensar el arte desde lo social ${ }^{101}$.

Fue durante la Segunda Internacional cuando se definió un marco político sobre el cual se desarrollaron los primeros historiadores del arte marxistas, como

92. MAUCLAIRE, Camille. "L'CEuvre sociale de l'art moderne. Les Beaux Arts». La Revue socialiste, octubre de 1901, pp. 421-435.

93. Herbert, Eugenia W. The Artist and Social Reform: France and Belgium 1885-1898. New Haven: Yale U. P., 1961

94. McWilliam, Neil; Méneux, Catherine y Ramos, Julie (dirs.). L'Art Social en France. De la Révolution à la Grande Guerre. Rennes: PUR, 2014. 2002.

95. Highmore, Ben. Everyday Life and Cultural Theory. An Introduction. Londres: Routledge,

96. PlekHANOV, Georgii V. Art and Social Life. Londres: Lawrence \& Wishart, 1953.

97. Mehring, Franz. The Lessing Legend. Nueva York: Marxist Critics Groups, 1938.

98. LifshitZ, Mikhail. The Philosophy of Art of Karl Marx. Nueva York: Critics Group, 1938.

99. Baxandall, Lee y Morawski, Stefan (eds.). A selection of writings. Marx E Engels on Literature \& Art. Nueva York: International General, 1974.

100. Hemingway, Andrew (ed.). Marxism and the History of Art. From William Morris to the New Left. Londres: Pluto Press, 2006.

101. McWilliam, Neil; Moréteau, Constance y Lamoureux, Joanne (dirs.). Histoires sociales de l'art. Une anthologie critique. París: Les presses du réel, 2016, 2 vols. 
Wilhelm Hausenstein y Eduard Fuchs ${ }^{102}$. Aunque la Revolución rusa obligó de nuevo a reorientar estas ideas, como mostrará la Tercera Internacional ${ }^{103}$, sobre todo, a medida que la vanguardia buscaba hacer la guerra al arte burgués. Momento en que se asistirá, por ejemplo, a la reorganización de las colecciones de los museos en función de la procedencia de clase de los artistas, definiendo un arte proletario ${ }^{104}$. Si bien el Partido se mostró permisivo hacia determinadas manifestaciones de la vanguardia, sin embargo, en 1928, con la inauguración del primer plan quinquenal de Stalin, la Unión Soviética vive un proceso de estalinización que se acentuará con el decreto emitido por el Comité Central de abril de 1932, en el que se llamará a la reconstrucción de la literatura y el arte. Este culminó la Revolución Cultural iniciada entre 1928 y 1931 que había buscado la proletarización intelectual frente a la inteligencia burguesa, imponiendo, finalmente, el realismo ${ }^{105}$. Momento que coincidirá con el reeplanteamiento del arte de vanguardia y la emergencia de los realismos ${ }^{106}$ en Europa, inscritos dentro de un proceso de politización artística generalizada ${ }^{107}$. La organización de escritores proletarios rusos (RAPP) defendió una concepción radical de la literatura realista, tanto formal como temáticamente, que convirtió el realismo socialista en la doctrina oficial a partir de Congreso de Escritores Soviéticos de $1934^{108}$, donde, si bien se buscó una reconciliación con la intelectualidad crítica ${ }^{109}$, sin embargo, se certificó la escisión dentro de esta corriente realista entre la oficial y la de autores como Georg Lukács y Mikhail Lifshits ${ }^{110}$.

Frente al materialismo dialéctico oficial, diversos intelectuales marxistas -como Lukács- plantearon una relectura de Marx influenciados por sus escritos de juventud de 1844, redescubiertos y publicados en 1932. En estos, la todavía influyente presencia de Hegel determinó la importancia otorgada a la cuestión de las

102. RumOlD, Rainer y WerCKMEISTER, O. K. (eds.). The Ideological Crisis of Expressionism: The Literary and Artistic German War Colony in Belgium, 1914-1918. Columbia: S. C. Camden House, 1990.

103. TAYLOR, Brandon. Art and Literature under the Bolsheviks. Londres: Pluto Press, 1992, 2 vols.

104. SCHMit, Theodor. "The Study of Art in USSR, 1917-1928». Parnassus, 1929, 1, 1, pp. 7-10.

105. FITZPATRICK, Sheila. The Cultural Front: Power and Culture in Revolutionary Russia. Ithaca: Cornell University Press, 1992.

106. RÉGNIER, Gérard (com.). Les Realismes, 1919-1939. Entre Révolution et Réaction. París: Centre Georges Pompidou, 1981.

107. AntLIFF, Mark. Inventing Bergson: Cultural Politics and the Parisian Avant-Garde. New Jersey: Princeton U. P., 1993.

108. FitZPATRICK, Sheila (ed.). Cultural Revolution in Russia, 1928-1931. Bloomington: Indiana University Press, 1984.

109. ERmolaEv, Herman. Soviet Literary Theories, 1917-1934. Berkeley: University of California Press, 1963.

110. GORKY, Maxim et al. Soviet Writers' Congress 1934. The Debate on Socialist Realism and Modernism in the Soviet Union. Londres: Lawrence \& Wishart, 1977. JAmES, Vaughan C. (ed.). Soviet Socialist Realism: Origins and Theory. Londres: Macmillan, 1973. 
representaciones culturales y al tema de la alienación del mundo moderno, lo que tendrá una gran acogida en el mundo intelectual europeo de los años 40. Todo ello favoreció una aproximación hegeliana a $\mathrm{Marx}^{111}$, como se observa en Marcuse o en Lefevbre, en el que las representaciones culturales, las formas de vida cotidiana y la alienación ocuparon un lugar preponderante frente a la infraestructura económica; condicionando, asimismo, las reflexiones sobre el realismo y sobre lo cotidiano, que ya habían sido tratadas de forma conjunta por el propio Lukács.

En relación a la Historia del Arte marxista habría que distinguir dos momentos ${ }^{112}$.

En primer lugar, el periodo que trascurre entre los años 30 y 40 del siglo xx, cuando algunos de los más conocidos historiadores del arte adscritos a la tradición marxista se acercan al movimiento comunista, como fue el caso de Antal, Hauser, Klingender, Schapiro o Max Raphael. Tanto Antal como Hausser formaron parte del movimiento intelectual húngaro conocido como Círculo de Domingo ${ }^{113}$, junto a Johannes Wilde, Karl Mannheim y Georg Lukács ${ }^{114}$, quien ejercía la principal influencia intelectual ${ }^{115}$. Esta interpretación marxista de Antal y Hauser influyó en la historiografía artísitica angloamericana, cuando, durante la guerra, ambos se trasladaron a vivir a Inglaterra. Por otro lado, en los Estados Unidos la crisis económica de los años 30 favoreció, igualmente, la defensa de un realismo instrumental entre los artistas ${ }^{116}$ y críticos de arte, aunque ciertos grupos de izquierda continuaron defendiendo lo moderno. Dentro de este contexto político y cultural complejo ${ }^{117}$ destacará la obra de Meyer Schapiro ${ }^{118}$ y su texto Nature of Abstract $A r t^{119}$, que marca un hito fundamental del debate artístico en Norteamérica ${ }^{120}$. En

111. Aron, Raymond. El marxismo de Marx. México, Siglo XXI, 2010, p. 20 y p. 592.

112. Wallach, Alan. "Marxism and art history». En Ollman, Bertell y Vernoff, Edward (eds.). The Left Academy. Marxist Scholarship on America Campuses. Nueva York: Praeger, 1984, vol. 2, pp. $25-53$.

113. Congdon, Lee. The Young Lukács. Londres: Chapel Hill, 1983, p. 118. Gluck, Mary. Georg Lukács and His Generation, 1900-1918. Cambridge: Harvard University Press, 1985, p. 11.

114. Arato, Andrew y Breines, Paul. The Young Lukács and the Origins of Western Marxism. Londres: Pluto Press, 1979. LÖwY, Michael. Georg Lukács: from Romanticism to Bolschevism. Londres: New Left Books, 1979.

115. Wessely, Anna. "Antal and Lukács: The Marxist Approach to the History of Art». New Hungarian Quarterly, 1979, 20, 73, pp. 114-125.

116. Hemingway, Andrew. Artist on the Left: American Artist and the Communist Mouvement, 1926-1956. New Haven: Yale University Press, 2002.

117. Denning, Michael. The Cultural Front: The Laboring of American Culture in the Twentieth Century. Londres: Verso, 1998.

118. Werckmeister, O. K. "Romanesque Art by Meyer Schapiro». Art Quarterly, 1979, 2, 2, pp. $211-218$

119. SCHAPIRO, Meyer. "Nature of Abstract Art». Marxist Quarterly, 1937, 1, pp. 78-97. HeminGWAY, Andrew. "Meyer Schapiro and Marxism in the 1930s". Oxford Art Journal, 1994, vol. 17, 1, pp. $13-29$.

120. PATTERSON, Jody. "Marxism et critique d'art: Meyer Shapiro et "la nature de l'art l'abstrait" . En McWilliam, Moréteau y Lamoureux. Histoires sociales de l'art..., vol. 1., pp. 145-155. 
él se opuso tanto a la ortodoxia marxista de la infraestructura económica, como a las vías de análisis formalistas que proponían Clement Greenberg y Alfred H. Barr, Jr., defendiendo la necesidad de inscribir la modernidad dentro de las problemáticas sociales del siglo XIX, cuestionando aquel relato del arte moderno en el que la abstracción era la culminación lógica e inevitable del arte europeo.

Entre 1935 y 1941 se asistió a un repliegue del marxismo, imponiéndose tras la guerra una visión formalista del arte en la que ocupará un lugar destacado la defensa de la abstracción realizada por parte de Greenberg y Barr, a través de varias exposiciones $^{121}$ donde se vehiculizaba un relato legitimador del orden establecido tras la guerra ${ }^{122}$. Así, desde mediados de los años 50 el Congreso por la libertad de la cultura $^{123}$-una organización anticomunista creada durante la Guerra Fría- desarrollará una larga ofensiva para orientar la Historia del Arte en un sentido formalista, ante la penetración de las ideas marxistas en el mundo de las artes ${ }^{124}$.

El segundo momento importante se producirá a partir de los años 60 y 70, cuando asistimos a una recuperación de las ideas marxistas, coincidiendo con un fenómeno de agitación social que de la calle pasará a las aulas universitarias. El aumento demográfico tras la guerra y la necesidad de su disciplinamiento fomentaron la proliferación de unas universidades que se abrieron a un sector cada vez más amplio de población, favoreciendo una sociedad estudiantil que criticaba la política internacional de potencias como los Estados Unidos, así como el marco de valores que parecía haberse impuesto durante la Guerra Fría, reivindicando un cambio. Todo ello favoreció, finalmente, la politización de la universidad y el resurgir de un marxismo condicionado en gran parte por la lectura de Lukács, la Escuela de Fráncfort y por Althusser, donde las cuestiones de las representaciones culturales y el tema de la alienación moderna, junto a las reivindicaciones de un arte social que debía retornar a lo cotidiano -donde parecía residir la revolución-, entretejieron finalmente un discurso complejo en el que se irá gestando una nueva lectura de la pintura de género, en paralelo al surgimiento de una nueva Historia del Arte.

Será en las universidades de finales de los años 60, valoradas como instituciones de reproducción de la ideología hegemónica, donde se propondrá una reflexión crítica sobre disciplinas tales como la Historia del Arte. Alemania fue uno de los primeros lugares donde se producirá no solo la agitación estudiantil de la universidad -que se extenderá a otros países occidentales-, sino, también, la redefinición de la Historia del Arte en torno a las ideas marxistas frente al formalismo anterior.

121. Wallach, Allan. Exhibiting Contradictions. Essay on the Art Museum in the United States. Boston: University of Massachusetts Press, 1998.

122. SAunders, F. S. La CIA y la guerra fría cultural. Madrid: Penguin Random House, 2013, pp. 172 y s., pp. 369 y s.

123. GRÉMION, Pierre. Intelligence de l'anticomunisme. Le Confrès pour la liberté de la culture à Paris (1950-1975). París: Fayard, 1995.

124. EGBERT, Donald Drew. Social Radicalism and the Arts. Nueva York: Alfred Knopf, 1970. 
En 1968 se crearon, por parte de un grupo de estudiantes y de jóvenes profesores (entre ellos Thomas Gaehtgens), la Ulmer Verein y la revista Kritische Berichte ${ }^{125}$, que pondrá el acento sobre las cuestiones culturales, a partir de los textos de la Escuela de Fráncfort. Asimismo, en Inglaterra se estaban desarrollando desde finales de los años 50 los Cultural Studies ${ }^{126}$, adscritos a los grupos sindicales. Estos buscaban analizar las profundas transformaciones que estaban teniendo lugar en una sociedad marcada por el consumo ${ }^{127}$. Afrontaban sus estudios mediante un enfoque variado y multidisciplinar, centrado en las representaciones culturales y en la alienación de las clases trabajadoras en las sociedades de consumo. La influencia de los pensadores franceses como Althusser, Barthes, Bourdieu o Lefebvre, así como la lectura del joven Marx realizada por Lukács o la Escuela de Fráncfort, les permitió alejarse de la ortodoxia marxista y, de este modo, la cultura no la comprendían ya como un simple reflejo de la infraestructura económica y de la lucha de clases, sino como un campo simbólico de luchas específicas por la hegemonía cultural, caracterizada asimismo por la resistencia. Si bien en Inglaterra los Cultural Studies otorgaban un lugar destacado al problema de las clases sociales, sin embargo, en los Estados Unidos predominará la cuestión de la representación cultural, favoreciendo el desplazamiento del interés hacia los imaginarios culturales, las comunidades y las minorias sociales.

Las reivindicaciones civiles y la agitación universitaria de finales de los 60 determinaron el progresivo interés de las universidades norteamericanas por dar respuesta a las reclamaciones sociales y a la diversidad cultural (dentro de una visión comercial de la universidad) ${ }^{128}$; lo que favoreció a partir de los años 70 la importanción del pensamiento europeo $-\mathrm{y}$, sobre todo, francés- ${ }^{129}$. Valorado por unos como una vía para superar la tradición marxista ${ }^{130}$ y por otros como una posible respuesta a los problemas identitarios y a las reivindicaciones de las

125. HAMMER-SCHENK, Harold et al. Kunstgeschichte gegen den Strich gebrüstet? 10 Jabre Ulmer Verein. 1968-1978. Hanover: Ulmer Verein für Kunst-und Kulturwissenschaften, 1979. WARNKE, Martin (ed.). Das Kunstwerk zwischen wissenschaft und Weltanschauung. Gütersloh: Bertelsmann Kunstverlag, 1970. Werckmeister, O. K. "Radical Art History». Art Journal, 1982, 42, 4, pp. 284-291. WerCKMEISTER, O. K. "The Turn from Marx to Warburg in West German Art History, 1968-1990». En Hemingway, Andrew (ed.). Marxism and the History of Art..., pp. 213-220.

126. GirouX, Henry y McLaren, Peter (eds.). Between Borders: Pedagogy and the Politics of Cultural Studies. Londres: Routledge, 1994. STEELE, Tom. The Emergence of Cultural Studies, 1945-1965: Cultural Politics, Adult Education and the English Question. Londres: Lawrence and Wishart, 1997. Aldned, Nannette y RYle, Martin (eds.). Teaching Culture: The Long Revolution in Cultural Studies. Leicester: NIASE, 1999. MATTELART, Armand y NEveU, Érik. Introduction aux Cultural Studies. París: La Découverte, 2003.

127. Dworkin, Dennis. Cultural Marxism in Postwar Britain. History of the New Left and the Origins of Cultural Studies. Durham: Duke University Press, 1997.

128. Turaine, Alain. Université et Société aux États-Unis. París: Seuil, 1972, p. 121.

129. Cusset, François. French Theory. Foucault, Derrida, Deleuze E Cie et les mutations de la vie intellectuelle aux États-Unies. París: La Découverte, 2003.

130. Ibid., p. 70. 
minorias, el pensamiento francés se puso al servicio de la mutación política acontecida en los Estados Unidos, donde las reivindicaciones civiles daban paso a las luchas identitarias por la hegemonía cultural. Asimismo, tal y como se mostró en el Coloquio Marxista de la Historia del Arte de Maburgo, de 1979, desde época temprana existió un estrecho diálogo entre los grupos marxistas europeos y angloamericanos. En los Estados Unidos la principal referencia será la universidad de UCLA, donde impartían clases David Kunzle y O. K. Werckmeister, y donde serán invitados T. J. Clark, Albert Boime o Nicos Hadjinicolaou.

En el mundo anglosajón la New Left y los Cultural Studies favorecieron nuevas aproximaciones a la Historia del Arte, donde la obra de Schapiro actuó de bisagra entre el marxismo de los años 30 y el de los 70, por ejemplo, en autores como T. J. Clark, Albert Boime, Robert L. Herbert, Thomas Crow, Guilbaut, etc. Aunque Clark no se identificaba con el marxismo dialéctico y prefirió hablar de Social History of $A r t^{131}$, incidiendo, precisamente, en la relación entre forma y contenido ideológico ${ }^{132}$. En Norteamérica la New Left y el arte estuvieron mucho más estrechamente vinculados a ciertos artistas militantes, así como al movimiento feminista, tal y como se reflejó con la formación de la New Art Association (NAA), en enero de 1970. Entre los historiadores del Arte que participan en la NAA estuvieron Carol Duncan, Edward Fry, Patricia Hills, Eunice Lipton, Linda Nochlin, D. Stephen Pepper o Alan Wallach. De este modo, en 1975, a las reivindicaciones del mundo artístico se unirán las reivindicaciones feministas, como reflejará de Artist Meeting for Cultural Change (AMCC). El mismo año Clark, Kunzle y Werckmeister decidieron inaugurar un encuentro titulado Marxism and Art History, dentro del encuentro anual del College Art Association de 1976, creando el Caucus for Marxism and Art History (posteriormente Caucus for Marxism and Art). Pero no encontrarán mucho éxito, pues las posturas más radicales hacia las instituciones y la enseñanza relacionada con el arte se venían dando del lado del feminismo, cuyas tesis habían culminado en 1972 con el Women's Caucus for Art ${ }^{133}$. Esta situación demostraba el estancamiento de las ideas marxistas que se tuvieron que redefinir dentro de nuevos campos emergentes como el feminismo ${ }^{134}$. Aunque algunos miembros de este movimiento no consideraban el marxismo adecuado a

131. Clark, T. J. "The Conditions of Artistic Creativity». Times Literary Supplement, 24 de mayo de 1974, pp. 561-562.

132. Clark, T. J. "Preliminary Arguments: Work of Art and Ideology". Papers Presented to the Marxism and Art History Session of the College Art Association Meeting in Chicago, February 1976. Los Ángeles: University of California, 1977.

133. Broude, Norma y D. Garrard, Mary. Feminism and Art History: Questioning the Litany. Nueva York: Harper \& Row, 1982.

134. CowARD, Rosalinda y Ellis, John. Language and Materialism: Developments in Semiology and Theory of Subject. Londres: Routledge and Kegan Paul, 1977. Pollock, Griselda. "Vision, Voice and Power: Feminist Art History and Marxism». En Pollock, Giselda. Vision and Difference: Feminity, Feminism and the Histoire of Art. Londres: Routledge, 1988 (publicado por vez primera en Block, 1982, n. ${ }^{\circ}$ 6). 
sus fines ${ }^{135}$, ya que tradicionalmente había obviado el papel de la mujer y de las cuestiones sexuales en sus reflexiones, y, de este modo, para Linda Nochlin, Carol Ducan o Griselda Pollock, era más importante el modelo del patriarcado que la noción de clase en la producción cultural. No obstante, a partir de la New Left y de los Cultural Studies estas minorias: sexuales, de género, raciales, etc., incorporaron el marxismo a sus propias reivindicaciones y luchas, favoreciendo una gran variedad de perspectivas dentro de la Historia del Arte, entre los años 80 y 90, que se aglutinarán bajo el término New Art History ${ }^{136}$; lo que produjo la desintegración de la disciplina ${ }^{137}$ y su cuestionamiento ${ }^{138}$. Es dentro de esta nueva Historia del Arte donde asistiremos a la vinculación entre la pintura de género, el realismo y lo cotidiano, construyendo unos modelos interpretativos ajenos a la Francia del siglo XVIII, determinados, en gran medida, por las luchas por la hegemonía cultural en los propios Estados Unidos ${ }^{139}$.

\section{LA PINTURA FRANCESA DEL SIGLO XVIII EN LA HISTORIOGRAFÍA ANGLOAMERICANA}

Una de las principales referencias de la historiografía angloamericana a la hora de acercarse al estudio de la pintura francesa del siglo XviII fue la obra de Antal, como señalaban las obras de Schapiro, Brown o Rosemblum, quienes reconocían la lectura del texto Clasicismo y Romanticismo ${ }^{140}$. Escrito a partir de una serie de artículos aparecidos entre 1935 y 1941 en The Burlington Magazine, Antal se centraba en la pintura francesa entre finales del siglo XVIII y principios del siglo XIX, siguiendo los análisis de Walter Friedländer, De David a Delacroix, de 1930, así como la obra de Plekhanov, Teatro y pintura en Francia en el siglo XVIII, de $1909^{141}$. Esta última había determinado la reordenación de la colección de pintura francesa del Museo del Hermitage, siguiendo una visión social de clase que influirá en Antal tras su visita al museo en $1932^{142}$.

Plekhanov había vivido en París a comienzos del siglo xx, donde había podido leer la producción historiográfica literaria de la Tercera República, de Lavisse,

135. Rowbotham, S.; Segal, L. y Wainwright, H. Beyond the Fragments: Feminism and the Making of Socialism. Londres: Merlin, 1979. Gouma-Peterson, Thalia y MatHEws, Patricia. "The Feminist Critique of Art History». Art Bulletin, 1987, pp. 327-357.

136. ReEs, A. L. y Borzello, Frances (eds.). The New Art History. London: Camden Press, 1986. Harris, Jonathan. The New Art History. A Critical Introduction. Londres: Routledge, 2001.

137. RifKin, Adrian. "Art's Histories». En ReES y BorZELlo. The New Art History..., pp. 157-163.

138. Belting, Hans. Das Ende der Kunstgeschichte? München: Deutscher Kunstverlag, 1983.

139. Rifkin, Adrian. "Can Gramsci save art history?». Block, 3, 1981, pp. 35-40.

140. ANTAL, Frederick. Clasicismo y Romanticismo. Madrid: Alberto Corazón, 1978.

141. Plekhanov, Georgii V. "French Drama and Painting of the Eighteenth-Century». En PleKHANOv, George V. Art and Society \& Others Papers in Historical Materialism. Nueva York: Oriole Editions, 1974.

142. ANTAL, Frederick. «Über Musee in der Sowjetunion (1932)». Kritische Berichte, 1976, 4, 2/3, pp. $5-13$ 
Lanson o Brunetière; así como la historiografía artística sobre el siglo XVIII, tanto de los frères Goncourt como los trabajos científicos universitarios de la naciente historia del arte dirigidos por Henry Lemonnier ${ }^{143}$. Plekhanov propuso en su artículo un modelo interpretativo sobre el arte francés, a partir de la burguesía, que daba inicio poniendo en paralelo las transformaciones en el teatro y las transformaciones en la pintura. Analizaba así el teatro popular y su vinculanción con la farsa -cuyo origen situaba en el Medievo-, y su sustitución por la tragedia con Louis XIII, que representaría las nuevas formas de la aristocracia. Plekhanov no solo enfatiza en su artículo esta tensión entre lo popular - la farsa- y lo aristocrático -la tragedia-, sino, también, el diálogo entre ambas, como plantearán posteriormente Brown o Crow. Subrayaba, asimismo, la aparente paradoja de cómo el surgimiento de un drama burgués a mediados del siglo XVIII, definido por su naturalismo y simplicidad, no produjo un cuestionamiento de las formas clásicas de la tragedia, sino, por el contrario, una acentuación de las mismas que caracterizará el periodo revolucionario. Si la transformación económica producida por el estallido de la burbuja de la Banca Law supuso para él un cuestionamiento de los valores aristocráticos y la emergencia de la burguesia, reflejado en el drama burgués a mediados de siglo, sin embargo, los espectadores y la nueva clase burguesa continuarán favoreciendo las formas clásicas. Para Plekhanov fue a mediados de siglo que se produjo el cuestionamiento del modelo trágico en pintura, como se reflejaría en la pintura de género de Greuze, quien abandonaría los valores voluptuosos del reinado de Louis XV a favor de los valores del drama burgués. Un cambio determinado por la influencia del sentimentalismo inglés y por las transformaciones burguesas que estaban teniendo lugar en Inglaterra, tal y como seañaló Hautecoeur ${ }^{144}$. No obstante, para Plekhanov fue la obra de David la que representó las aspiraciones burguesas durante la Revolución, transformando los símbolos clásicos. Este texto ponía las bases de un modelo interpretativo sobre la pintura francesa del siglo XVIII que, además de ser recogido por Antal, quien establecerá igualmente una relación entre el «naturalismo» o la pintura de género y la burguesía, constituirá un lugar común de los estudios norteamericanos marxistas como los de Milton Brown The Painting of the French Revolution ${ }^{145}$ -alumno de Friedländer- o de Thomas Crow.

Tras la Segunda Guerra Mundial, si bien las aproximaciones sociales al arte disminuyeron, sin embargo, habría que destacar la publicación en 1951 de la obra de Hauser Historia Social de la Literatura y el Arte, traducida al inglés en 1954. Profundamente contestada ${ }^{146}$, constituyó, sin embargo, una referencia insoslayable

143. THERRIEN, Lyne. L’histoire de l'art en France. París: CTHS, 1998, pp. 284-285.

144. Hautecoeur, Louis. "Le Sentimentalisme dans la peinture Française, de Greuze a David». Gazette des Beaux-Arts, 106, 51 Année, 1909.

145. Brown, Milton W. The Painting of the French Revolution. Nueva York: Critics Group, 1938, p. 68 .

146. Orwicz, Michael R. "Critical discourse in the formation of a Social History of Art. Angloamerican response to Arnold Hauser». Art Journal, 1985, 8, 2, pp. 52-62. 
para las aproximaciones sociales al arte en el mundo anglosajón -aunque muchas de ellas no la citasen-. En su libro IV, Rococó, Clasicismo y Romanticismo, Hauser elaboró una visión de conjunto sobre la pintura francesa del siglo XVIII retomando los temas principales de la obra de Plekhanov, así como de los estudios franceses de la Tercera República, poniendo el acento sobre las cuestiones de la lucha entre representaciones culturales, lo que le permitirá enfatizar la ruptura del arte rococó ${ }^{147}$, que se desvinculará de la clase social (tal y como luego observaremos en Crow). Para Hauser fue a mediados del siglo XVIII cuando penetró en el arte el "naturalismo» (tras el que subyace el problema del realismo) frente al modelo clásico anterior, que se convierte en el auténtico dinamizador del arte:

Ahora se descubre por vez primera el valor de la verdad artística como arma en la lucha social. Ahora se es consciente por primera vez de que la reproducción fiel de los hechos conduce por sí misma a la disolución de los prejuicios sociales y a la abolición de la injusticia, de que aquellos que luchan por la justicia no tienen que temer la verdad en ninguna de sus formas, y de que, en una palabra, existe una cierta concordancia entre la idea de la verdad artística y la de la justicia social. Ahora surgió aquella alianza tan conocida en el siglo XIX entre el radicalismo y el naturalismo, aquella solidaridad que los elementos progresistas sintieron que existía entre ellos y los naturalistas ${ }^{148}$.

En la década de los años 40 del siglo xx asistimos a la emergencia de un nuevo estilo: el neoclasicismo ${ }^{149}$, que venía a ser una respuesta desde el formalismo al supuesto surgimiento de un gusto por lo clásico en la década de los 60 del siglo XVIII, como ya habían subrayado Bertrand ${ }^{150}$ o Locquin ${ }^{151}$. El término tuvo su principal acogida en el periodo de entreguerras, cuando diversos movimientos comenzaron a cuestionar el arte de vanguardia, defendiendo una vuelta a lo clásico, al orden, a la forma, etc. Instantes en que Mario Praz escribe sus primeros textos sobre el gusto neoclásico que atraerán la atención del mundo anglosajón, especialmente tras la guerra (David Irwin, English Neoclassical Art: Studies in Inspiration and Taste de 1966; Hugh Honour, Neoclasicismo de 1968; y, sobre todo, la exposición de 1972 The Age of Neoclasicism). Estas obras trataban de analizar las transformaciones producidas en la segunda mitad del siglo XVIII a través de un nuevo estilo que permitía culminar la aspiración inglesa de reivindicar su importancia en el arte europeo, frente a una Historia del Arte dominada hasta entonces

147. Hauser, Arnold. Histoire Social de la Literatura y el Arte. II. Madrid: Guadarrama, 1964, p. 13.

148. Ibid., pp. 97-98.

149. Michel, Régis (com.). Le Beau Idéal, ou l'art du concept. Paris: RMN, 1989. Craske, Matthew. Art in Europe, 1700-1830: A History of the Visual Arts in an Era of Unprecedented Urban Economic. Oxford: Oxford University Press, 1997.

150. BERTRAND, Louis. La fin du classicisme et le retour à l'antique. Paris: Hachette, 1897.

151. LoCQuin, Jean. La peinture d'Histoire en France de 1747 à 1785. Paris: H. Laurens, 1912. 
por franceses y alemanes ${ }^{152}$. En este contexto, Rosenblum afrontó en los 60 el análisis de estas transformaciones del arte francés de finales del siglo XVIII a partir de la cuestión de lo clásico, ofreciendo una doble perspectiva en la que se daba cabida tanto al modelo formalista de su maestro Friedländer -creando nuevos géneros dentro del neoclasicismo-, como al modelo social, citando a Antal y a Brown y poniendo el acento sobre la clase burguesa y la influencia inglesa como dinamizadores de las transformaciones ${ }^{153}$.

En los años 70 asistimos a una emergencia de las ideas marxistas en el ámbito universitario, determinando el resurgir del interés por el romanticismo y, sobre todo, por el realismo, como reflejarán las obras de Clark. De hecho, el director del museo de Cleveland Sherman E. Lee, a propósito de la exposición The Realist Tradition: French Painting and Drawing, 1830-1900 de 1981, se preguntaba sobre el porqué de esta eclosión del interés por la cuestión del realismo en los últimos años ${ }^{154}$, esto es, entre finales de los años 70 y los años $80^{155}$. A pesar de lo cual, el realismo continuó sin ser el protagonista en los estudios sobre la pintura francesa del siglo XVIII, ya que las principales obras habían enfatizado la cuestión de lo clásico, así como las perspectivas políticas ${ }^{156}$. No obstante, en la obra fundamental de Thomas Crow, Painters and Public Life in Eighteenth-Century Paris, de 1985, lo popular y sus variantes -como el realismo-, a través del rococó, volvía a adquirir predominancia como dinamizador del arte, pero siempre en diálogo con lo clásico, como había señalado Plekhanov.

Formado en el entorno de Clark y Boime, y en la línea espiritual de Schapiro $^{157}$, Crow buscó borrar las influencias marxistas para abrirse a las nuevas interpretaciones culturales de la New Left ${ }^{158}$; lo que se observa en el uso de una noción más amplia de clase y en su análisis de las representaciones culturales en conflicto. Su texto se construyó, pues, a partir de dos tradiciones marxistas muy claras. Por un lado, la corriente proveniente de Plekhanov, Antal, Brown o Hauser -heredera, a su vez, de los estudios republicanos de la Tercera República

152. PASsini, Michela. La fabrique de l'art national. Le nationalisme et les origines de l'bistoire de l'art en France et en Allemagne 1870-1933. París: FMSH, 2012.

153. Rosenblum, Robert. Transformaciones en el arte de finales del siglo XVIII. Madrid: Taurus, 1986, p. 53.

154. LeE, Sherman E. «Foreword». En WeISBERG, The Realist Tradition..., p. XIII.

155. Solomon-Godeau, Abigail. "Realism Revisited». En D'Souca, Aruna (ed.). Self and History: Essays in Honor of Linda Nochlin. Nueva York: Tahmes \& Hudson, 2001, pp. 69-75.

156. DowD, David. Pegeant-Master of the Republic: Jacques-Louis David and the French Revolution. Lincoln: University of Nebraska Press, 1948. LEITH, James A. The idea of Art as Propaganda in France, 1750-1799. Toronto: University of Toronto, 1965.

157. Crow, Thomas. «Modernism and Mass Culture». En Buchloh, Benjamin; Guilbaut, Serge y Solkin, David (eds.). Modernism and Modernity: The Vancouver Conference Papers. Halifax: The Press of the Nova Scotia-College of Art and Design, 1983.

158. McWilliam, Neil. «Vers une histoire de l'histoire sociale de l'art». En McWilliam, MorétEaU y LAmoureuX. Histoires sociales de l'art..., vol. 1, p. 27. 
francesa-, enfatizando los diálogos entre cultura popular y alta cultura, vinculando formas artísticas, grupos sociales e imaginarios culturales en conflicto -reflejados para él en la formación del rococó-. Por otro lado, y a partir de la cuestión del espacio público y la opinión, fueron claras sus deudas con la Escuela de Fráncfort y, especialmente, con el texto de Habermas Historia y crítica de la opinión pública, de 1962. En su obra ocupó un lugar predominante la cuestión de la burguesía, cuyo auge y conciencia irán en paralelo a la definición de un espacio público donde luchó -junto con otros grupos- por definir un arte que le representase y donde se sientiese reconocida en sus aspiraciones políticas y sociales (dando entrada a uno de los principios fundamentales del realismo). Es en ese espacio público donde se producirá para Crow la hibridación entre alta y baja cultura y, finalmente, la apropiación de la alta cultura por parte de la clase emergente, adscribiéndose al clasicismo.

Si bien la pintura francesa de género había sido señalada por diversos estudios, ya desde el siglo XIX, como un hito fundamental para analizar el cambio social del dieciocho, sin embargo, al incidirse siempre sobre la ruptura revolucionaria y sobre la obra de David, se continuó poniendo el acento sobre el problema de lo clásico. A excepción de algunas tesis doctorales ${ }^{159}$, no será hasta finales de los años 90 cuando esta pintura adquiera un lugar preponderante en los estudios sobre el periodo. Dentro de un contexto caracterizado por el resurgir de las cuestiones de lo cotidiano ${ }^{160}$ (en 1991 se traducirá al inglés el primer volumen de Critique de la vie quotidienne de Lefebvre de 1947); por la hegemonía de los Cultural Studies (ante el auge de las reivindicaciones identitarias de la sociedad americana, donde la lectura de la obra de Lefebvre había sido una constante desde los años 70); y por el éxito de la obra de Michel de Certeau ${ }^{161}$ (quien fue invitado en los años 80 por la universidad de California) ${ }^{162}$. Asimismo, se asistió al resurgir de la fenonomenología, a medida que la fiebre estructuralista se apaciguó, lo que animó a pensar de nuevo la subjetividad humana y el fenómeno de la realidad, donde lo cotidiano ocupaba un lugar fundamental, tal y como reflejaba la obra de Lefebvre, quien había buscado en los años 60 incorporar a su análisis la fenomenología de Merleau-Ponty, como había hecho ya Lukács respecto a Husserl.

Si bien la reflexión sobre la cotidianidad había aparecido en la crítica literaria, por ejemplo, en el Surrealismo; en la sociología, de la mano de autores como Simmel; o en la filosofía, en Benjamin, Heidegger o Lukács; sin embargo, no será hasta la obra de Lefebvre cuando se producirá su redefinición clara, precisamente,

159. MCPHERSON, Heather Ann. Some Aspects of Genre Painting and its Popularity in EighteenthCentury France. Ph.D. diss. University of Washington, 1982.

160. Sheringham, Michael. Everyday Life. Theories and Practices from Surrealisme to the Present. Oxford: Oxford U.P., 2009, p. 3.

161. Certeau, Michel de. L'invention du quotidien. París: Gallimard, 1980.

162. Cusset. French Theory..., p. 148. 
a partir de ciertas lecturas de Heidegger y Lukács ${ }^{163}$. En oposición a ambos, Lefebvre buscó definir lo cotidiano de forma positiva ${ }^{164}$, superando la oposición entre realismo y cotidianidad articulada en Lukács. Para lo cual partió de la conciencia desventurada de Hegel, así como de los textos de Marx de 1844 sobre la ideología, la mistificación y la alienación; planteando, finalmente, un estrecho vínculo entre alienación y lo cotidiano, comprendiendo este último como una vía para superar aquella. Los textos del joven Marx sobre las representaciones culturales analizados por Lukács se convirtieron, por tanto, en el punto de partida de la reflexión sobre lo cotidiano tanto en los seguidores del filósofo húngaro, como Agnes Heller, como entre los intelectuales marxistas franceses como Lefebvre. Lo cotidiano aparecerá, así, en la Escuela de Annales, por ejemplo, en Lucien Febvre tras su noción de outillage mental o en Fernand Braudel en Civilisation matérielle, économie et capitalisme $^{165}$ de 1969. Todo ello reveló un vivo debate en Francia a finales de los 60 en torno a lo banal y lo cotidiano, dentro de una sociedad francesa que estaba cambiando profundamente ${ }^{166}$, tal y como se puso de manifiesto entre aquellos que estaban reflexionando sobre las formas de la sociedad de consumo, como los Situacionistas, Castoriadis, Morin, Barthes o Baudrillard. Ideas que rápidamente tuvieron su acogida en el mundo anglosajón a través de los Cultural Studies, quienes estaban interesados también en la sociedad de consumo y en la alienación, situando a lo cotidiano como el nuevo territorio para la resistencia y la lucha.

Entre la exposición de 1997: Intimate Encounters, y la exposición de 2003: The Age of Watteau..., lo cotidiano se convirtió en una constante de la reflexión universitaria norteamericana; lo que coincidirá nuevamente con la traducción al inglés, en 2002, del segundo volumen de Critique de la vie quotidienne de Lefebvre, publicado en 1961. Ambas exposiciones proponían, por tanto, una revisión de la pintura de género francesa del siglo XVIII en función de las teorías de lo cotidiano recogidas por los Cultural Studies, incorporando a la Historia del Arte -y concretamente a la pintura francesa- aquel desplazamiento acontecido en diversas disciplinas en los años 30, desde el realismo a lo cotidiano. Textos posteriores demostraron la consolidación de este nuevo modelo interpretativo ${ }^{167}$ que convertía la pintura de género francesa del siglo XVIII en una producción marginal, a través de la cual se descubría no tanto una lucha de clases sino una lucha entre representaciones, así como una resistencia al poder establecido y al discurso hegemónico

163. Guterman, Norbet y Lefebvre, Henri. La Conscience mystifiée. París: Gallimard, 1936.

164. Trebitsch, Michel. «Preface». En LEFEBvre, Henri. Critique of Everyday Life. Londres: Verso, 1991.

165. BRAUDEL, Fernand (dir.). Civilisation matérielle, économie et capitalisme, XVe-XVIII siècle 1. Les Structures du quotidien - 2. Les Jeux de l'échange - 3. Le Temps du monde. París: Armand Colin, 1979.

166. Ross, Kristin. Fast Cars, Clean Bodies: Descolonization and the Reordering of French Culture. Cambridge: MIT U. P., 1996.

167. Anderman, Barbara J. Petits sujets, grandes machines. Critical Battles over Gentre Painting in France 1660-1780. Ph.D diss. Rutgers, The State University of New York, 2000. 
de la Academia ${ }^{168}$, revelándose, finalmente, a través de ella aquellas fuerzas que configuraban las representaciones culturales y que dinamizaban la historia.

These eighteenth-century representations of the everyday tend to offer more questions than answers about the nature of lived experience. Many carry within them significant discourses of economic and political power, controversial dialogues about gender and race, and cultural critiques. Their value lies [...] no in their "truth", but in their ability to eloquently illuminate the desires, preocupations, and shared illusions of their creators and consumers, making in matter less wether the "everyday» as a homogenous experience exists now, or ever existed, beyond its power as shared ideal ${ }^{169}$.

A través de esta pintura se buscó penetrar en la producción cultural de la época: en el amor, en la familia, en el rol de la mujer, en las formas de consumo, en las formas de deseo, etc.; ya que reflejaba un «dialogue between the constantly shifting modes of popular culture and established forms of artistic expression» ${ }^{170}$. Una tesis que fundamentó, asimismo, el symposium que tuvo lugar en Washington a propósito de la exposición The Age of Watteau..., titulado French Genre Painting in the Eighteenth Century, en el cual, como reconoce Philip Conisbee, se certificó la consolidación de los modelos de análisis propuestos por la New Art History. Conisbee parecía alejarse así de sus propios posicionamientos de los años 80, planteados en Painting in Eighteenth Century France, donde había definido la noción de género según la tradición historiográfica derivada de André Félibien:

The position of genre painting in the eighteenth century is especially interesting, as it was essentially modern in its rejection of the elevated subject matter of history and mythology in favor on the interpretation of everyday life, albeit with varysing degrees of realistic observation or imaginative invention ${ }^{171}$.

Podemos concluir el presente artículo señalando que desde los 90 una parte de la historiografía artística angloamericana construyó un modelo interpretativo sobre la pintura de género francesa del siglo XVIII a partir de la noción de lo cotidiano, que no era más que una reactualización de los imaginarios y discusiones - políticas y estéticas- del siglo XIX y XX, adscritas al marxismo. Animaban, pues, a mirar aquella pintura en función de problemáticas y principios ajenos a los debates poetológicos, políticos y sociales, de la Francia del siglo XVIII, lo que dificulta actualmente su correcta comprensión.

168. Cavanaugh, Alden (ed.). Performing the "Everyday». The Culture of Genre in the Eighteenth Century. Newark: University of Delaware Press, 2007, p. 13.

169. Cavanaugh, Performing the "Everyday»..., p. 15.

170. RuB, Thimothy. "Foreword». En RAND. Intimates Encounters..., p. VII.

171. Conisbee, Philip. "Introduction». En CONISBEe, Philip (ed.). French Genre Painting in the Eighteenth Century. Washington, New Haven: Center for Advanced Study in the Visual Arts, Yale University Press, 2007, p. 10. 
